\title{
Cartan Connections on Lie Groupoids and their Integrability
}

Anthony D. BLAOM

10 Huruhi Road, Waiheke Island, New Zealand

E-mail: anthony.blaom@gmail.com

Received May 19, 2016, in final form December 02, 2016; Published online December 07, 2016

http://dx.doi.org/10.3842/SIGMA.2016.114

\begin{abstract}
A multiplicatively closed, horizontal $n$-plane field $D$ on a Lie groupoid $G$ over $M$ generalizes to intransitive geometry the classical notion of a Cartan connection. The infinitesimalization of the connection $D$ is a Cartan connection $\nabla$ on the Lie algebroid of $G$, a notion already studied elsewhere by the author. It is shown that $\nabla$ may be regarded as infinitesimal parallel translation in the groupoid $G$ along $D$. From this follows a proof that $D$ defines a pseudoaction generating a pseudogroup of transformations on $M$ precisely when the curvature of $\nabla$ vanishes. A byproduct of this analysis is a detailed description of multiplication in the groupoid $J^{1} G$ of one-jets of bisections of $G$.
\end{abstract}

Key words: Cartan connection; Lie algebroid; Lie groupoid

2010 Mathematics Subject Classification: 53C05; 58H05; 53C07

\section{Introduction}

This article describes a generalization of classical Cartan connections using the language of Lie groupoids, with which the reader is assumed to have some familiarity. We recommend the introduction given in [7]. For more detail, see [12, 17, 23].

The present article provides some detail missing from our work published on related matters $[3,4,5,6]$ and is more technical than those works. Applications of the Lie groupoid approach to Cartan connections will be given elsewhere.

\subsection{Cartan connections on Lie groupoids}

Let $G$ be a Lie groupoid over a smooth connected manifold $M$ and $J^{1} G$ the corresponding Lie groupoid of one-jets of local bisections of $G$. Then a right-inverse $S: G \rightarrow J^{1} G$ for the canonical projection $J^{1} G \rightarrow G$ determines a $n$-plane field $D \subset T G$ on $G$, where $n=\operatorname{dim} M$. If $S: G \rightarrow J^{1} G$ is additionally a morphism of Lie groupoids, we call $D$ (or $S$ ) a Cartan connection on $G[4,6]$. In the general terminology of [16], $S$ is a unital flat cleavage for the Lie groupoid fibration $J^{1} G \rightarrow G$. Or, if we view the tangent bundle $T G$ as a Lie groupoid over $T M$, then $D \subset T G$ should be multiplicatively closed in the sense of $[14,25]$.

Cartan connections on Lie groupoids are the global analogues of the Cartan connections on Lie algebroids first introduced in [3] - here called infinitesimal Cartan connections - and studied further in $[4,5]$. Infinitesimal Cartan connections also appear in a new approach to Cartan geometries introduced in the recent monograph [15] (where they are called Blaom connections).

Cartan connections in various incarnations have been central to the study of finite-type geometric structures, and to the geometric approach to studying overdetermined systems of partial differential equations. The first example can be found in Ėlie Cartan's seminal paper [9]. In the present article a classical Cartan connection is a certain one-form on a principal $H$-bundle $P$ taking values in a vector space $V$ with $\operatorname{dim} V=\operatorname{dim} P$, as first formulated by Ehresmann [18]. 
See Section 2.1 for the definition and [26] for a nice introduction. Our first result, proven in Sections 2 and 3.3, is a correspondence between classical Cartan connections and Cartan connections on transitive Lie groupoids:

Theorem 1.1. Every classical Cartan connection $\omega$ on a principal $H$-bundle $P$ determines a canonical Cartan connection $S^{\omega}$ on the corresponding gauge groupoid $G_{P}=(P \times P) / H$. Conversely, every Cartan connection on a transitive Lie groupoid $G$ coincides with $S^{\omega}$, for some classical Cartan connection $\omega$, after the identification $G \cong G_{P}$ determined by a choice of source-fibre $P \subset G$.

It should be noted here that our notion of a classical Cartan connection is, by default, stripped of any 'model data' because such data plays no role at all in the above correspondence' ${ }^{1}$.

It was shown already in [3] that every classical Cartan connection on a principal bundle $P$ determines an infinitesimal Cartan connection on the associated Atiyah Lie algebroid $T P / H$.

\section{$1.2 \quad$ Examples}

To illustrate the utility of Cartan connections in a simple example, suppose $M$ is a Riemannian manifold, and let $M \times M$ be the pair groupoid. Regard elements of $J^{1}(M \times M)$ as one-jets of local transformations of $M$ and let $G \subset J^{1}(M \times M)$ be the subgroupoid of all one-jets $J_{m}^{1} \phi$ for which the tangent map $T_{m} \phi: T_{m} M \rightarrow T_{\phi(m)} M$ is an isometry. Then it can be shown (see the appendix) that $G$ carries a canonical Cartan connection $D$ whose significance is this: The firstorder extension $b=J^{1} \phi$ of every local isometry $\phi$ of $M$ is a local bisection of $G$ integrating $D$ (i.e., $D$ is tangent to $b$, when $b$ is viewed as a submanifold of $G$ ). And, conversely, every $n$ dimensional integral manifold of $D$ is locally a local bisection of $G$ arising as the extension of a local isometry $\phi$. In other words, the local isometries are in one-to-one correspondence with the sufficiently localised $n$-dimensional integral manifolds of $D$. In particular, obstructions to the involutivity (integrability) of $D$ amount to obstructions to the existence of local isometries of $M$.

The main result of the present article, formulated in Section 1.4 below, concerns the infinitesimal (Lie algebroid) version of obstructions to the involutivity of a Cartan connection.

The prototype of an involutive Cartan connection $D$ is the one associated with the action of a Lie group $G_{0}$ on $M$. In this case one takes $G$ to be the action groupoid $G_{0} \times M$ (transitive only if the action is transitive) and defines a Cartan connection as follows: $S(g, m)$ is the one-jet at $m$ of the constant bisection $m^{\prime} \mapsto\left(g, m^{\prime}\right)$.

More examples of Cartan connections on Lie groupoids will be given elsewhere.

\subsection{Infinitesimal Cartan connections}

Infinitesimalizing a Cartan connection, viewed as a Lie groupoid morphism $S: G \rightarrow J^{1} G$, we obtain a splitting $s: \mathfrak{g} \rightarrow J^{1} \mathfrak{g}$ of the exact sequence

$$
0 \longrightarrow T^{*} M \otimes \mathfrak{g} \longrightarrow J^{1} \mathfrak{g} \longrightarrow \mathfrak{g} \longrightarrow 0 .
$$

Here $J^{1} \mathfrak{g}$ denotes the Lie algebroid of one-jets of sections of the Lie algebroid $\mathfrak{g}$ of $G$. (A natural identification of the Lie algebroid of $J^{1} G$ with $J^{1} \mathfrak{g}$ is recalled in Section 3.1 below.) In the category of vector bundles, splittings $s$ of the above sequence are in one-to-one correspondence with Koszul connections $\nabla$ on $\mathfrak{g}$; this correspondence is given by

$$
s X=J^{1} X+\nabla X, \quad X \in \Gamma(\mathfrak{g}) .
$$

(According to our sign conventions, the inclusion $T^{*} M \otimes \mathfrak{g} \rightarrow J^{1} \mathfrak{g}$ induces the following map on sections: $d f \otimes X \mapsto f J^{1} X-J^{1}(f X)$.) When $s: \mathfrak{g} \rightarrow J^{1} \mathfrak{g}$ is a morphism of Lie algebroids, as is the

\footnotetext{
${ }^{1}$ Model data is part of Ehresmann's original formulation.
} 
case here, then $\nabla$ is called a Cartan connection on $\mathfrak{g}$ [3], or an infinitesimal Cartan connection when we want to distinguish it from the corresponding global notion defined in Section 1.1. As usual, the curvature of $\nabla$ is defined by

$$
\operatorname{curv} \nabla(X, Y) Z=\nabla_{X} \nabla_{Y} Z-\nabla_{Y} \nabla_{X} Z-\nabla_{[X, Y]} Z
$$

and $\nabla$ is flat if $\operatorname{curv} \nabla=0$.

\subsection{The main theorem}

The central question to be addressed in the present article is this:

To what extent are obstructions to the involutivity of a Cartan connection D encoded in the corresponding infinitesimal Cartan connection $\nabla$ ?

To answer this question, let $D$ be a Cartan connection on a Lie groupoid $G$ and let $G^{D} \subset G$ denote the set of points through which there exist $n$-dimensional integral manifolds of $D, n=$ $\operatorname{rank} D=\operatorname{dim} M$.

\section{Theorem 1.2.}

(1) If a Cartan connection $D$ on a Lie groupoid $G$ over $M$ is involutive in some neighborhood $V$ of $M$ then the corresponding infinitesimal Cartan connection $\nabla$ is flat.

(2) $G^{D}$ is a wide subgroupoid of $G$ and, when $\nabla$ is flat, a union of connected components of $G$ containing the source-connected component $G^{0}$ of $G$.

The proof of this theorem and its connection to other work is discussed in Sections 1.6 and 1.7 below.

\subsection{Pseudoactions}

If $\nabla$ above is flat then, replacing $G$ with a union of connected components, $D$ is tangent to a foliation $\mathcal{F}$ on $G$. We now axiomatize the sense in which $\mathcal{F}$ is compatible with the multiplicative structure of $G$, and how it accordingly generates a pseudogroup of local transformations on $M$ (the pseudogroup of isometries of $M$ in the case of a maximally symmetric Riemannian manifold).

Let $G$ be a Lie groupoid and call an immersed submanifold $\Sigma \subset G$ a pseudotransformation if the restrictions to $\Sigma$ of the groupoid's source and target maps are local diffeomorphisms. A pseudoaction [6] on $G$ is any smooth foliation $\mathcal{F}$ on $G$ such that:

(1) The leaves of $\mathcal{F}$ are pseudotransformations.

(2) $\mathcal{F}$ is multiplicatively closed.

To define what is meant in (2), regard local bisections of $G$ as immersed submanifolds and let $\widehat{\mathcal{F}}$ denote the collection of all local bisections that intersect each leaf of $\mathcal{F}$ in an open subset. Let $\widehat{G}$ denote the collection of all local bisections of $G$, this being a groupoid over the collection of all open subsets of $M$. Then condition (2) is the requirement that $\widehat{\mathcal{F}} \subset \widehat{G}$ be a subgroupoid.

Given a pseudoaction $\mathcal{F}$ of $G$ on $M$, each element $b \in \widehat{\mathcal{F}}$ defines a local transformation $\phi_{b}$ of $M$. Let $\operatorname{pseud}(\mathcal{F})$ denote the set of all local transformations $\psi: U \rightarrow V$ of $M$ that are locally of this form; that is, for every $m \in U$, there exists a neighborhood $U^{\prime} \subset U$ of $m$ such that $\left.\psi\right|_{U^{\prime}}=\phi_{b}$ for some for some $b \in \widehat{\mathcal{F}}$. Then, by $(2), \operatorname{pseud}(\mathcal{F})$ is a pseudogroup.

A pseudoaction generalizes the ordinary action of a Lie group $G_{0}$ on $M$ : Take $G$ to be the corresponding action groupoid $G_{0} \times M$ and $\mathcal{F}$ the foliation whose leaves are $\{g\} \times M, g \in G_{0}$. For more examples see [6], where the following elementary observation is established: 
Proposition 1.3. An arbitrary foliation $\mathcal{F}$ on $G$ is a pseudoaction if and only if its tangent $n$-plane field $D$ is a Cartan connection on $G$.

Under an additional completeness hypothesis, a pseudoaction becomes an ordinary Lie group action, with possibly a monodromy 'twist' [6].

An immediate corollary to Theorem 1.2 can now be stated as follows:

Corollary 1.4. Assume $G$ is source-connected. Then a Cartan connection $D$ is tangent to a pseudoaction $\mathcal{F}$ on $G$ if and only if the corresponding infinitesimal Cartan connection $\nabla$ is flat.

In practice, the pseudogroup generated by a pseudoaction $\mathcal{F}$ represents a pseudogroup of symmetries, the Riemannian example mentioned in Section 1.2 being a case in point. We consequently make the following interpretation:

A Cartan connection on a Lie groupoid is a (possibly intransitive) 'symmetry deformed by curvature'.

\subsection{Connections to other work}

Theorem 1.2(1) and the above corollary already follow from a result of Salazar [25, Theorem 6.4.1] (see also [14]) for arbitrary 'multiplicatively closed' distributions on a Lie groupoid, of which the $n$-plane field $D$ defined by a Cartan connection is a special case. The independently obtained proof of Theorem 1.2 given in Section 5 is based on: (i) A detailed analysis of the multiplicative structure of $J^{1} G$, given in Section 3; and (ii) A characterization of the infinitesimal connection $\nabla$ as infinitesimal parallel translation in the Lie groupoid $G$ along $D$, described in Section 4. We expect both analyses will be of some independent interest.

In Salazar's approach, one identifies the obstruction to the involutivity of $D$ with a Lie groupoid cocycle $c$, whose infinitesimalization $\kappa$, a Lie algebroid cocycle, is seen to vanish when $\nabla$ is flat. Van Est's isomorphism between Lie groupoid and Lie algebroid cohomology, generalized to Lie groupoids and algebroids [11], then implies that the cohomology class $[c]$ must vanish. To show $c$ itself vanishes requires a further topological argument.

Although the basic idea behind Salazar's proof and the present one are reasonably straightforward, flushing out the details requires some work in both cases. Salazar's result has the advantage that it applies in greater generality; we hope that our proof sheds light on the special case important to the theory of Cartan connections.

As far as we know, the first statement (without proof) of Corollary 1.4 appears in [4, Appendix A].

By Corollary 1.4, the infinitesimalization of a pseudoaction is a flat Cartan connection on a Lie algebroid, also called by us a twisted Lie algebra action, for reasons explained in [6]. Analogues of the so-called fundamental theorems, Lie I, Lie II and Lie III, for Lie groups and Lie algebras, are established for pseudoactions and twisted Lie algebra actions in [6], to which the present article can be regarded as a prequel.

The modern redevelopment of Cartan geometries along lines promoted here, and in our other works cited above, has several aspects warranting further investigation. We briefly discuss two here.

First, there have yet to appear many interesting applications of the theory to intransitive geometric structures (structures that Ehresmann's formulation cannot handle). One reason for this may be the focus of current research on intransitive structures of infinite type, a subject far richer than its transitive counterpart. These include: Dirac structures [10], which generalize Poisson, presymplectic, and foliated structures; generalized complex structures [19]; and Jacobi structures [20, 21, 22] which simultaneously generalize Poisson and contact structures (see also [13]). However, add extra structure, or perturb the underlying 'integrability condition', 
and these structures generally become finite-type structures which presumably admit a Cartan geometry interpretation. Moreover, adding extra structure, such as a compatible metric, may shed light on the underlying infinite-type structure, in the same way that equipping a Riemann surface with a metric sheds light on the underlying topology of the surface.

Secondly, quite a large number of examples of transitive geometric structures - including conformal, CR, and projective structures - are of so-called parabolic type, a notion cast very decidedly in Ehresmann's formulation of a Cartan geometry (including model data) [8]. It would be worthwhile finding an axiomatization of parabolic geometries in terms of Lie groupoids or Lie algebroids. An intriguing observation in this direction is that the Atiyah algebroid of a parabolic geometry is also a twisted Courant algebroid [1, 2, 27].

\subsection{Multiplication in $J^{1} G$ and parallel translation}

In making a proof of Theorem 1.2, we have been led to make a detailed investigation of the multiplicative structure of $J^{1} G$, which is given in Section 3. While not hard to guess at this description - at the level of the bisection group, it is a semidirect product - in writing out details the following technical problem arises: Given two paths in a Lie groupoid $g(t), h(t) \in G$ such that the product $g(t) h(t)$ is always defined, compute the time derivative of the product $g(t) h(t)$ in terms of the time derivatives of the individual factors. In the category of Lie groups, a trivial application of the chain rule solves the problem, but this application rests on the fact that $g(t) h(s)$ is defined for $s \neq t$, which needn't be the case for arbitrary Lie groupoids. The solution adopted here is to parallel translate the factors using an appropriate connection $D$ on the groupoid.

As we show in Section 4, if one infinitesimalizes the parallel translation associated with a Cartan connection $D$ on $G$, then one recovers the corresponding infinitesimal Cartan connection $\nabla$ on $\mathfrak{g}$ defined in Section 1.3 above. This alternative characterization of $\nabla$ is the key to our proof of Theorem 1.2.

\section{Classical Cartan connections}

We now construct, for each classical Cartan connection $\omega$ on a principal $H$-bundle $P$, a Cartan connection $S^{\omega}$ on the gauge groupoid $(P \times P) / H$. A converse construction, completing the proof of Theorem 1.1, is postponed until Section 3.3. In Section 2.4 we describe the infinitesimalization of $S^{\omega}$, a Cartan connection $\nabla^{\omega}$ on the Atiyah Lie algebroid $T P / H$ of $P$.

In this section only, $G$ denotes a Lie group, and not a Lie groupoid.

\subsection{Classical Cartan connections}

Let $H$ be a Lie group with Lie algebra $\mathfrak{h}$, let $\pi: P \rightarrow M$ be a right principal $H$-bundle, and suppose $P$ is equipped with a parallelism $\omega$. Then $\omega$ is called a Cartan connection on $P$ if:

(1) The infinitesimal generators $\xi^{\dagger}, \xi \in \mathfrak{h}$, of $H$ are $\omega$-parallel.

(2) The space of $\omega$-parallel vector fields is invariant under the canonical right action of $H$ on the space of all vector fields (the action by pullback).

In this article, to distinguish this use of the term from the one given previously, we will call $\omega$ a classical Cartan connection.

We may understand the parallelism $\omega$ as a one-form on $P$ taking values in some vector space $V$, and whose restriction to each tangent space is an isomorphism onto $V$. Since we may identify $V$ with the space of $\omega$-parallel vector fields (the ones on which $\omega$ is constant) condition (1) realizes $\mathfrak{h}$ as a subpace of $V$ and we obtain the tautology

(3) $\omega\left(\xi^{\dagger}(p)\right)=\xi$, for all $\xi \in \mathfrak{h}$ and $p \in P$. 
On the other hand, condition (2) means that $V$ becomes a right representation of $H$, and this action extends the adjoint action in the sense that $\xi h=\operatorname{Ad}_{h^{-1}} \xi$ whenever $\xi \in \mathfrak{h}$. Tautologically, then, we also have the equivariance condition

(4) $\omega(v h)=\omega(v) h$ for all $h \in H, v \in T P$.

More commonly, one prescribes a vector space $V$, an inclusion $\mathfrak{h} \subset V$ and a right representation of $H$ on $V$ extending the adjoint action, and defines a Cartan connection as a parallelism $\omega: T P \rightarrow V$ satisfying (3) and (4). However, in this case it is easy to see that (3) and (4) imply (1) and (2) and that consequently the two formulations are equivalent.

For example, if we take $H$ to be a closed subgroup of some Lie group $G$, acting on $G$ from the right, then we may take $V=\mathfrak{g}$, where $\mathfrak{g}$ is the Lie algebra of $G$, and take $H$ to act on $\mathfrak{g}$ by the adjoint action: $\xi h:=\operatorname{Ad}_{h^{-1}} \xi, \xi \in \mathfrak{g}$. Then the left-invariant Mauer-Cartan form $\omega$ on $G$ is a Cartan connection on the principal bundle $G \rightarrow G / H$ satisfying the well-known Mauer-Cartan equation $d \omega-\frac{1}{2} \omega \wedge \omega=0$, where a wedge implies a contraction using the bracket on $\mathfrak{g}^{2}{ }^{2}$

\subsection{Cartan connections with model data}

Generalizing the previous example, we may consider Cartan connections on an arbitrary principal $H$-bundle $P \rightarrow M$ for which $V=\mathfrak{g}$ and $H$ acts via restricted adjoint action. One refers to $G / H$ as the homogeneous model and the extra data (the Lie group $G$ and the embedding $H \subset G$ ) as model data. With model data prescribed, one defines the curvature of $\omega$ by $\Omega=d \omega-\frac{1}{2} \omega \wedge \omega$, where the wedge again implies a contraction using the bracket on $\mathfrak{g}$ (part of the model data!). If $\Omega=0$, then $M$ can be identified, at least locally, with the homogeneous model $G / H$ [26, Theorem 5.3]. More generally, this leads one to regard $M$ with the given data as a homogeneous space 'deformed by curvature'.

Two points must be emphasised when comparing this 'deformed by curvature' interpretation with the analogous interpretation of Cartan connections on Lie groupoids: First, the homogeneous model $G / H$ is specified a priori; in particular, if $M$ happens to be a different homogeneous space, then generally $\Omega \neq 0$. Secondly, only transitive symmetry can follow from the 'flatness' $(\Omega=0)$ of a classical Cartan connection.

In fact, since every classical Cartan connection determines a Cartan connection on a Lie groupoid, there is already a notion of curvature which does not depend on the prescription of model data, but its interpretation is different: in the transitive case this curvature vanishes when $M$ is locally isomorphic to some homogeneous space, not necessarily a model fixed beforehand. See also [5] and Remark 2.5.

The literature devoted to classical Cartan connections which include model data is large. The reader is referred to the book [26] for an introduction, and to [8] for a more recent survey. Note that an important work in which model data is not prescribed is Morimoto's construction of Cartan connections for filtered manifolds [24].

\subsection{Cartan connections from classical Cartan connections}

The notion of a parallelism on a smooth manifold $P$ can be reformulated using Lie groupoid language as follows: Let $S: P \times P \rightarrow J^{1}(P \times P)$ be any Cartan connection on the pair groupoid $P \times P$ in the sense of Section 1.1. Note that elements of $J^{1}(P \times P)$ may be understood as onejets of local transformations $\phi: U \rightarrow V$ of $P$. Now $J^{1}(P \times P)$, and hence the Lie subgroupoid $G=S(P \times P)$, acts canonically on the tangent bundle $T P$. Call a vector field on $P$ parallel if it is $G$-invariant, and denote the $n$-dimensional space of parallel vector fields by $V, n=\operatorname{dim} P$.

\footnotetext{
${ }^{2}$ We define brackets on Lie algebras and Lie algebroids using right-invariant vector fields.
} 
The tautological map $\omega: T P \rightarrow V$ satisfying $\omega(X(m))=X$ for all $X \in V$ is a parallelism, in sense of Section 2.1, and evidently all parallelisms arise in this way.

Now suppose $\pi: P \rightarrow M$ is a right principal $H$-bundle and let $H$ act on $J^{1}(P \times P)$ from the right by Lie groupoid morphisms according to

$$
\left(J_{p}^{1} \phi\right) h=J_{p h}^{1}\left(R_{h} \circ \phi \circ R_{h^{-1}}\right),
$$

where $R_{h}(p):=p h$, and let $J^{1}(P \times P)^{\mathfrak{h}} \subset J^{1}(P \times P)$ denote the subset consisting of one-jets of local transformations $\phi$ of $P$ that are infinitesimally $H$-equivariant, which means $\phi^{*} \xi^{\dagger}=\xi^{\dagger}$, for all $\xi$ in $\mathfrak{h}$. Then $J^{1}(P \times P)^{\mathfrak{h}}$ is a transitive $H$-invariant Lie subgroupoid of $J^{1}(P \times P)$, as is readily verified.

Proposition 2.1. The parallelism $\omega: T P \rightarrow V$ defined by a Cartan connection $S$ on $P \times P$ is a classical Cartan connection on $P$ if and only if $S$ satisfies the following conditions:

(1) $S: P \times P \rightarrow J^{1}(P \times P)$ takes values in $J^{1}(P \times P)^{\mathfrak{h}}$.

(2) $S(q h, p h)=S(p, q) h$ for $p, q \in P$ and $h \in H$ (H-equivariance).

The easy proof is left to the reader.

The key observation that allows one to drop a Cartan connection $S$ on $P \times P$ to a Cartan connection on the gauge groupoid $(P \times P) / H$ is the following:

Lemma 2.2. As Lie groupoids over $M$, we have $J^{1}((P \times P) / H) \cong J^{1}(P \times P)^{\mathfrak{h}} / H$.

The following comments will help the reader follow the proof:

(3) Our convention is to regard the source map of the pair groupoid $P \times P$ as $(q, p) \mapsto p$.

(4) Given an element $J_{p}^{1} \phi \in J^{1}(P \times P)^{\mathfrak{h}}$ represented by some infinitesimally $H$-equivariant $\phi$, the maps $R_{h} \circ \phi$ and $\phi \circ R_{h}$ have the same germ at $p$, for all $h \in H$ in some neighborhood of the identity. This is because infinitesimal $H$-equivariance implies local $H$-equivariance. (Consequently, for all $H$ sufficiently close to the identity, we simply have $\left(J_{p}^{1} \phi\right) h=J_{p h}^{1} \phi$.)

(5) Viewing a bisection of $(P \times P) / H$ as a right inverse for the source projection $(q, p) \bmod H \mapsto$ $\pi(p)$, the local triviality of the bundle $\pi: P \rightarrow M$ allows us to write such a bisection in the form $b(m)=(q(m), p(m)) \bmod H$, for some smooth local right-inverse $m \mapsto p(m)$ of $\pi: P \rightarrow M$, and some smooth map $m \mapsto q(m)$, where the composite $\pi \circ q$ is a diffeomorphism onto its image.

Proof of Lemma 2.2. A map $A: J^{1}(P \times P)^{\mathfrak{h}} / H \rightarrow J^{1}((P \times P) / H)$ is defined as follows. Given an element $J_{p_{0}}^{1} \phi \bmod H \in J^{1}(P \times P)^{\mathfrak{h}} / H$ represented by some infinitesimally $H$-equivariant local transformation $\phi$ of $P$, first choose a local right-inverse $m \mapsto p(m)$ for $\pi: P \rightarrow M$ satisfying $p\left(m_{0}\right)=p_{0}$. Then we put

$$
A\left(J_{p_{0}}^{1} \phi \bmod H\right)=J_{m_{0}}^{1} b,
$$

where $m_{0}=\pi\left(p_{0}\right)$ and $b$ is the local bisection of $(P \times P) / H$ defined by

$$
b(m)=(\phi(p(m)), p(m)) \bmod H .
$$

Let $m \mapsto \bar{p}(m)$ be a second local right-inverse for $\pi$ satisfying $\bar{p}\left(m_{0}\right)=p_{0}$. Then $\bar{p}(m)=$ $p(m) h(m)$, for some smooth map $m \mapsto h(m) \in H$ satisfying $h\left(m_{0}\right)=$ id. For all $m$ sufficiently close to $m_{0}$ we have $\phi(p(m) h(m))=\phi(p(m)) h(m)$, by $(4)$, in which case $(\phi(\bar{p}(m)), \bar{p}(m))$ mod $H$ $=(\phi(p(m)), p(m)) \bmod H$, which shows that the definition of $b$ is (up to germ equivalence at $\left.m_{0}\right)$ independent of the choice of $m \mapsto p(m)$. 
To show that the definition of $A\left(J_{p_{0}}^{1} \phi \bmod H\right)$ is independent of the choice of representative $J_{p_{0}}^{1} \phi$, it is enough to observe: (i) if $J_{\bar{p}_{0}}^{1} \bar{\phi}$ is a second representative then the one-jet of $\bar{\phi}$ at $\bar{p}_{0}$ coincides with the one-jet of $R_{h} \circ \phi \circ R_{h^{-1}}$, where $h \in H$ is determined by $\bar{p}_{0}=p_{0} h$; and (ii) in that case a local right-inverse $m \mapsto \bar{p}(m)$ for $\pi$ satisfying $\bar{p}\left(m_{0}\right)=\bar{p}_{0}$ is given by $\bar{p}(m)=p(m) h$, where $p(m)$ is as above.

We leave it to the reader to show that $A$ is a Lie groupoid morphism. To show $A$ is injective, let $J_{m_{0}}^{1} b \bmod H$ be an element of $J^{1}((P \times P) / H)$ with $b$ as in (5). Since each point of $P$ in a neighborhood of $p_{0}:=p\left(m_{0}\right)$ is of the form $p(m) h$, for some uniquely determined $h \in H$ and $m \in M$, a local transformation $\phi$ of $P$ is well-defined by $\phi(p(m) h)=q(m) h$. Then $\phi$ is $H$-equivariant by construction, and hence infinitesimally $H$-equivariant, and we have $A\left(J_{p_{0}}^{1} \phi \bmod H\right)=J_{m_{0}}^{1} b$.

The proof that $A$ is injective is no more difficult and is omitted.

Now let $\omega$ be a classical Cartan connection, understood as the parallelism defined by a Cartan connection $S$ on $P \times P$ satisfying (1) and (2). By the lemma, a Cartan connection $S^{\omega}$ on the quotient $(P \times P) / H$ is the same thing as a Lie groupoid morphism

$$
S^{\omega}:(P \times P) / H \rightarrow J^{1}(P \times P)^{\mathfrak{h}} / H
$$

furnishing a right-inverse for the canonical projection $J^{1}(P \times P)^{\mathfrak{h}} / H \rightarrow(P \times P) / H$. By the preceding proposition, such a connection is well-defined by

$$
S^{\omega}((p, q) \bmod H)=S(p, q) \bmod H, \quad p, q \in P .
$$

\subsection{Infinitesimalization of a classical Cartan connection}

Let $\omega: P \rightarrow V$ be a classical Cartan connection on a principal $H$-bundle as above. Then the infinitesimalization of the Cartan connection $S^{\omega}$ is a Cartan connection $\nabla^{\omega}$ on the Atiyah Lie algebroid $T P / H$. To show how $\nabla^{\omega}$ is related to $\omega$, let $\bar{\nabla}$ denote the infinitesimal parallelism determined by $\omega$, i.e., the flat Koszul connection on $T P$ for which the notions of $\omega$-parallel and $\bar{\nabla}$-parallel coincide; $\bar{\nabla}$ is characterised by the identity

$$
\omega\left(\bar{\nabla}_{X} Y\right)=d(\omega(Y))(X), \quad X, Y \in \Gamma(T P) .
$$

Lemma 2.3. Let $X$ and $Y$ be $H$-invariant vector fields on $P$. Then:

(1) $\bar{\nabla}_{X} Y$ is $H$-invariant.

(2) $\bar{\nabla}_{X} Y=[X, Y]$ whenever $Y$ is vertical.

Proof. Since $\omega$ is $H$-equivariant, a vector field on $P$ is $H$-invariant if and only if the $V$-valued function $\omega(X)$ is $H$-equivariant. Given this, and using the fact that the time- $t$ flow map of an $H$-invariant vector field commutes with the action of $H$, it is not hard to prove (1). To prove (2) it suffices, by (2.1), to prove that the Lie derivative $\mathcal{L}_{X} \omega$ vanishes on vertical vectors. To this end, let $\xi \in \mathfrak{h}$ and $p \in P$ be given. Then, denoting the time- $t$ flow map of $X$ by $\Phi_{X}^{t}$, we compute

$$
\begin{aligned}
\left(\mathcal{L}_{X} \omega\right)\left(\xi^{\dagger}(p)\right) & =\left.\frac{d}{d t} \omega\left(\left.T \Phi_{X}^{t} \cdot \frac{d}{d s} p \exp (s \xi)\right|_{s=0}\right)\right|_{t=0} \\
& =\left.\frac{d}{d t} \omega\left(\left.\frac{d}{d s} \Phi_{X}^{t}(p \exp (s \xi))\right|_{s=0}\right)\right|_{t=0} \\
& =\left.\frac{d}{d t} \omega\left(\left.\frac{d}{d s} \Phi_{X}^{t}(p) \exp (s \xi)\right|_{s=0}\right)\right|_{t=0} \\
& =\left.\frac{d}{d t} \omega\left(\xi^{\dagger}\left(\Phi_{X}^{t}(p)\right)\right)\right|_{t=0}=\left.\frac{d}{d t} \xi\right|_{t=0}=0 .
\end{aligned}
$$

The third equality holds because the time- $t$ flow map of $X$ commutes with the action of $H$. 
Now the space of $H$-invariant vector fields on $P$ is in one-to-one correspondence with the sections of the Atiyah Lie algebroid $T P / H$. By part (1) of the lemma, the flat $T P$-connection $\bar{\nabla}$ therefore drops to a representation $\bar{\nabla}^{\omega}$ of the Lie algebroid $T P / H$ on itself. According to part (2) of the lemma, we have, for sections $X, Y$ of $T P / H$

$$
\bar{\nabla}_{X}^{\omega} Y=[X, Y]
$$

whenever $\# Y=0$. Here \# denotes the anchor of $T P / H$ and $[\cdot, \cdot]$ the canonical bracket on its sections. It follows that there is a $T M$-connection $\nabla^{\omega}$ on $T P / H$ defined implicitly by

$$
\bar{\nabla}_{X}^{\omega} Y=\nabla_{\# Y}^{\omega} X+[X, Y]
$$

The proof of the following is left as an exercise for the reader:

Proposition 2.4. The connection $\nabla^{\omega}$ is the infinitesimalization of the Cartan connection $S^{\omega}$.

In particular, $\nabla^{\omega}$ is an infinitesimal Cartan connection, a fact already observed in [3].

Remark 2.5. As a final remark, we observe that if we prescribe model data and define $\Omega=$ $d \omega-\frac{1}{2} \omega \wedge \omega$, then $\nabla^{\omega}$ is flat - implying $S^{\omega}$ defines a pseudoaction on a union of connected components of $(P \times P) / H-$ precisely when $\bar{\nabla} \Omega=0$ [3, Theorem $\mathrm{C}]$, a condition weaker than the vanishing of $\Omega$.

\section{The multiplicative structure of $J^{1} G$}

Let $G$ be a Lie groupoid. The Lie algebroid of $J^{1} G$ can be identified with $J^{1} \mathfrak{g}$, where $\mathfrak{g}$ denotes the Lie algebroid of $G$ (see Section 3.1 below). The canonical exact sequence,

$$
0 \longrightarrow T^{*} M \otimes \mathfrak{g} \longrightarrow J^{1} \mathfrak{g} \longrightarrow \mathfrak{g} \longrightarrow 0
$$

may be regarded as the derivative of a natural sequence of groupoid morphisms,

$$
\operatorname{Aut}(T M, \mathfrak{g}) \hookrightarrow J^{1} G \rightarrow G .
$$

Here $\operatorname{Aut}(T M, \mathfrak{g})$ is a certain open neighborhood of the zero-section of $T^{*} M \otimes \mathfrak{g}$, defined in Section 3.4 below; if $G=M \times M$, then $\operatorname{Aut}(T M, \mathfrak{g})=\operatorname{Aut}(T M)$.

Corresponding to (3.1) is an exact sequence of section spaces which splits canonically, leading to an identification,

$$
\Gamma\left(J^{1} \mathfrak{g}\right) \cong \Gamma(\mathfrak{g}) \oplus \Gamma\left(T^{*} M \otimes \mathfrak{g}\right)
$$

Under this identification, the Lie bracket on $\Gamma\left(J^{1} \mathfrak{g}\right)$ is a semidirect product [3].

In this section we establish the global analogue of this result, namely a semidirect product structure,

$$
B\left(J^{1} G\right) \cong B(G) \times B(\operatorname{Aut}(T M, \mathfrak{g})),
$$

where $B(\cdot)$ denotes the group of global bisections.

Just as a choice of Cartan connection $\nabla$ on $\mathfrak{g}$ determines an identification

$$
J^{1} \mathfrak{g} \cong \mathfrak{g} \oplus\left(T^{*} M \otimes \mathfrak{g}\right)
$$

and an associated semi-direct product structure for $J^{1} \mathfrak{g}$, so a choice of Cartan connection on $G$ determines a semi-direct product structure for $J^{1} G$. While we shall provide a direct demonstration of this fact, the reader may like to interpret the existence of the semi-direct product 
structure as a consequence of the fact that $J^{1} G \rightarrow G$ is Lie groupoid fibration, and $S$ a unital flat cleavage for it [23, Theorem 2.5.3] (see also [16, Theorem 2.2.3]). In particular, $S$ determines a representation of $G$ on the kernel $J_{M}^{1} G$ which defines the semi-direct product structure.

The non-trivial part of our proof amounts to finding an explicit formula for the representation of $G$ on $J_{M}^{1} G$ using the adjoint representation of $J^{1} G$ on $\mathfrak{g}$ (see Section 3.2 below) and our concrete model Aut $(T M, \mathfrak{g})$ of $J_{M}^{1} G$. This description rests on a detailed analysis of multiplication in $J^{1} G$. To formulate our claims in detail, which we do in Section 3.5, will require some preparation.

\subsection{Representing elements of $J^{1} G$ and its Lie algebra}

There are three useful representations of an element of $J^{1} G$. Formally, an element of $J^{1} G$ is a one-jet at some $m \in M$ of a local bisection $b: U \rightarrow G$ of $G$. (It is our convention to regard local bisections as locally defined right-inverses for the source projection $\alpha: G \rightarrow M$.) If $g=b(m)$, then the tangent map $\mu:=T_{m} b$ is a linear map from $T_{m} M$ to $T_{g} G$ satisfying:

(1) $T_{m} \alpha \circ \mu=\mathrm{id}_{T_{m} M}$, and

(2) $T_{m} \beta \circ \mu: T_{m} M \rightarrow T_{m} M$ is invertible.

Conversely, for any $g \in G$ with $\alpha(g)=m$, any linear map $\mu: T_{m} M \rightarrow T_{g} G$ satisfying (1) and (2), is the tangent map of some local bisection $b: U \rightarrow G$ whose one-jet at $m \in M$ is independent of the particular choice of $b$. We are therefore entitled to identity elements of $J^{1} G$ with linear maps $T_{m} M \rightarrow T_{g} G$ with $\alpha(g)=m$ and satisfying (1) and (2), and will do so by default in the sequel.

Finally, each $\mu: T_{m} M \rightarrow T_{g} G$ as above may be identified with its image, a subspace of $T_{g} G$ that is simultaneously a complement for the tangent space at $g \in G$ to the fibre of the source projection $\alpha: G \rightarrow M$ through $g$, and the fibre of the target projection $\beta: G \rightarrow M$ through $g$; and all such 'simultaneous complements' may be realised in this way.

Let $\mathfrak{g}:=\mathcal{L}(G)$ denote the abstract Lie algebroid of $G$. Our convention is to regard an element of $\mathfrak{g}$ as a vector tangent at some $m \in M \subset G$ to a fibre of the source projection $\alpha: G \rightarrow M$. Let $J^{1} \mathfrak{g}$ denote the vector bundle of one-jets of sections of $\mathfrak{g}$. Then there is a natural isomorphism $\theta: \mathcal{L}\left(J^{1} G\right) \rightarrow J^{1} \mathfrak{g}$ whose inverse is described as follows: Let $J_{m}^{1} X \in J^{1} \mathfrak{g}$ be given. Then $\theta^{-1}\left(J_{m}^{1} X\right)$ will be a vector tangent to $J^{1} G$ at the identity element $T_{m} \iota_{M}: T_{m} M \rightarrow T_{m} M$ of $J^{1} G$ (with $\iota_{M}: M \rightarrow G$ denoting the inclusion) and so may be viewed as an equivalence class of paths in $J^{1} G$ passing through $T_{m} \iota_{M}$. Specifically,

$$
\theta^{-1}\left(J_{m}^{1} X\right)=\left.\frac{d}{d t} T_{m}\left(\Phi_{X}^{t} \circ \iota_{M}\right)\right|_{t=0},
$$

where $X^{\mathrm{R}}$ denotes the corresponding right-invariant vector field on $G$ and $t \mapsto \Phi_{X^{\mathrm{R}}}^{t}$ its flow.

\subsection{The adjoint representation}

The adjoint representation of a Lie group $G$ is a representation of $G$ on its Lie algebra. More generally, for each Lie groupoid $G$, one has a god-given representation of $J^{1} G$ on the Lie algebroid $\mathfrak{g}$ of $G$, which we also call the adjoint representation; it is defined as follows: Let $\mu \in J_{g}^{1} G$ be given, where $g \in G$ begins at $m \in M$ and ends at $m^{\prime} \in M$, and let $\left.X \in \mathfrak{g}\right|_{m}$. First, notice that we may use $\mu$ to lift $X$, viewed as a vector tangent to the source-fibre at $m$, to a vector $X^{\prime}$ tangent to the same source-fibre at $g$ :

$$
X^{\prime}=\mu(\# X)-T L_{g} \cdot T I \cdot X
$$


Here \# denotes the anchor, $I: G \rightarrow G$ inversion and $L_{g}: G \rightarrow G$ left-multiplication by $g$. (One has, incidentally, $T I \cdot X=\# X-X$, for all $X \in \mathfrak{g}$.) Then we define

$$
\operatorname{Ad}_{\mu} X:=T R_{g^{-1}} \cdot X^{\prime}=T R_{g^{-1}} \cdot\left(\mu(\# X)-T L_{g} \cdot T I \cdot X\right),
$$

where $R_{g}$ is right-multiplication by $g$. Notice that if $\# X=0$ (always true if $G$ is a Lie group) then this collapses to the familiar

$$
\operatorname{Ad}_{\mu} X=T\left(R_{g^{-1}} \circ L_{g}\right) \cdot X .
$$

Additionally, there is a natural representation of $J^{1} G$ on $T M$, with respect to which the anchor \#: $\mathfrak{g} \rightarrow T M$ is equivariant; this action, also denoted Ad, is given by

$$
\operatorname{Ad}_{\mu} v:=T \beta \cdot \mu(v), \quad v \in T_{m} M,
$$

where $\beta: G \rightarrow M$ is the target projection.

Finally, the two representations just defined induce a representation of $J^{1} G$ on $T^{*} M \otimes \mathfrak{g}$ needed later:

$$
\left(\operatorname{Ad}_{\mu} \phi\right) v=\operatorname{Ad}_{\mu}\left(\phi \operatorname{Ad}_{\mu}^{-1} v\right),\left.\quad \phi \in\left(T^{*} M \otimes \mathfrak{g}\right)\right|_{m} .
$$

For the sake of completeness, we add the following observation:

Remark 3.1. The adjoint representation amounts to a Lie groupoid morphism

$$
\mathrm{Ad}: \quad J^{1} G \rightarrow \mathrm{GL}(\mathfrak{g}),
$$

where $\operatorname{GL}(\mathfrak{g})$ is the frame groupoid of $\mathfrak{g}$. Infinitesimalizing, we obtain a Lie algebroid morphism ad: $J^{1} \mathfrak{g} \rightarrow \mathfrak{g l}(\mathfrak{g})$ coinciding with the adjoint representation of $J^{1} \mathfrak{g}$ on $\mathfrak{g}$, defined by

$$
\operatorname{add}_{J^{1} X} Y=[X, Y] \text {. }
$$

See, e.g., [3]. Here we are identifying the abstract Lie algebroid $\mathfrak{g l}(\mathfrak{g})$ of $\operatorname{GL}(\mathfrak{g})$ with the Lie algebroid of derivations on $\mathfrak{g}$ according to the following convention: Given an element of the abstract Lie algebroid represented by a path $t \mapsto \phi_{t} \in \mathrm{GL}(\mathfrak{g})$ with $\phi_{0}$ the identity on $\left.\mathfrak{g}\right|_{m}$ and $\phi_{t}$ an isomorphism from $\left.\mathfrak{g}\right|_{m}$ to $\left.\mathfrak{g}\right|_{m_{t}}$, for some path $t \mapsto m_{t} \in M$, the corresponding derivation $\partial$ is defined by

$$
\partial Y=\left.\frac{d}{d t} \phi_{t}^{-1} Y\left(m_{t}\right)\right|_{t=0}, \quad Y \in \Gamma(\mathfrak{g}) .
$$

\subsection{Recovering a classical Cartan connection}

We pause our development to show how a Cartan connection $S: G \rightarrow J^{1} G$ on a transitive Lie groupoid $G$ determines a classical Cartan connection $\omega$ on the source-fibre $P$ over an arbitrary fixed point $m_{0} \in M$. Recall that $P$ is a left principal $H$-bundle, where $H$ is the group of arrows of $G$ simultaneously beginning at terminating at $m_{0}$.

From the Cartan connection $S$ we obtain a representation of $G$ on its Lie algebroid $\mathfrak{g}: g \xi=$ $\operatorname{Ad}_{S(g)} \xi$. This is indeed a representation because $S: G \rightarrow J^{1} G$ is a Lie groupoid morphism. Letting $V=\left.\mathfrak{g}\right|_{m_{0}}$, we define $\omega: T P \rightarrow V$ by $\omega(v)=g^{-1}\left(T R_{g^{-1}} \cdot v\right)$, where $g \in P$ is the basepoint of $v \in T P$. One readily verifies axioms (3) and (4) of Section 2.1 defining a classical Cartan connection. Under the identification $G \cong(P \times P) / H$ the Cartan connection $S$ coincides with $S^{\omega}$, as defined in Section 2.3, as the reader is invited to check. This completes the proof of Theorem 1.1 . 


\subsection{A linear model of the kernel of $J^{1} G \rightarrow G$}

Let $J_{M}^{1} G \subset J^{1} G$ denote the pre-image of $M \subset G$ under the natural projection $J^{1} G \rightarrow G$; $J_{M}^{1} G$ is a totally intransitive Lie groupoid. We now define a certain open neighborhood of the zero-section of $T^{*} M \otimes \mathfrak{g}$, denoted Aut $(T M, \mathfrak{g})$, that serves as a model of $J_{M}^{1} G$.

For each $\phi \in T^{*} M \otimes \mathfrak{g}$ with base-point $m \in M$, define $\phi^{T M} \in T^{*} M \otimes T M$ by

$$
\phi^{T M} v=v-\# \phi v .
$$

Then, writing $\phi^{T M} \in \operatorname{Aut}(T M)$ if $\phi^{T M}: T_{m} M \rightarrow T_{m} M$ is invertible, the set

$$
\operatorname{Aut}(T M, \mathfrak{g}):=\left\{\phi \in T^{*} M \otimes \mathfrak{g} \mid \phi^{T M} \in \operatorname{Aut}(T M)\right\}
$$

is an open neighborhood of the zero-section of $T^{*} M \otimes \mathfrak{g}$. Moreover, $\operatorname{Aut}(T M, \mathfrak{g})$ becomes a totally intransitive Lie groupoid over $M$, with the zero-section becoming the set of identity elements, if we define multiplication by

$$
\psi \phi=\psi+\phi-\psi \circ \# \circ \phi=\phi+\psi \circ \phi^{T M}
$$

and inversion by

$$
\phi \mapsto-\phi \circ\left(\phi^{T M}\right)^{-1}
$$

With respect to this extra structure, the map

$$
\phi \mapsto \phi^{T M}: \operatorname{Aut}(T M, \mathfrak{g}) \rightarrow \operatorname{Aut}(T M)
$$

is a groupoid morphism (in this case, just a fibre-wise group homomorphism) with commutative kernel. In other words, for each $m \in M$, Aut $\left.(T M, \mathfrak{g})\right|_{m}$ is simply a commutative extension of the image of $\phi \mapsto \phi^{T M}$, a subgroup of $\left.\operatorname{Aut}(T M)\right|_{m}$.

Finally, note that in addition to the natural representation of $\operatorname{Aut}(T M, \mathfrak{g})$ on $T M$ given by $\phi \cdot v=\phi^{T M} v$, we have representation of $\operatorname{Aut}(T M, \mathfrak{g})$ on $\mathfrak{g}$ given by

$$
\phi \cdot X=\phi^{\mathfrak{g}} X:=X-\phi \# X, \quad X \in \mathfrak{g} .
$$

With respect to these actions of $\operatorname{Aut}(T M, \mathfrak{g})$ on $\mathfrak{g}$ and $T M$, the anchor \# is equivariant:

$$
\# \phi^{\mathfrak{g}} X=\phi^{T M} \# X .
$$

According to Theorem 3.3(1) below, we have $\operatorname{Aut}(T M, \mathfrak{g}) \cong J_{M}^{1} G$ as Lie groupoids.

\subsection{Multiplication and inversion in $J^{1} G$}

Inversion in $J^{1} G$ is straight-forward to describe. We leave the proof of the following to the reader:

Proposition 3.2 (inversion in $J^{1} G$ ). Suppose $g \in G$ begins at $m \in M$ and ends at $m^{\prime} \in M$. Then, for any $\mu \in J_{g}^{1} G$ the inverse $\mu^{-1} \in J_{g^{-1}}^{1} G$ is given, as a map $T_{m^{\prime}} M \rightarrow T_{g} G$, by

$$
\mu^{-1}(v)=T I \cdot \mu\left(\operatorname{Ad}_{\mu}^{-1} v\right), \quad v \in T_{m^{\prime}} M
$$

Here $I: G \rightarrow G$ denote inversion in $G$, and $T I: T G \rightarrow T G$ its tangent map. 
We now state our results for multiplication. First, recall that the Lie groupoid $\operatorname{Aut}(T M, \mathfrak{g})$ is totally intransitive; its bisections coincide with its sections, as an open neighborhood of the zero-section of the vector bundle $T^{*} M \otimes \mathfrak{g}$. We have seen that the adjoint representation induces an action of $J^{1} G$ on $T^{*} M \otimes \mathfrak{g}$. It is not hard to show that $\operatorname{Aut}(T M, \mathfrak{g}) \subset T^{*} M \otimes \mathfrak{g}$ is invariant under this action, and we obtain an action of the bisection group $B(G)$ on $B(\operatorname{Aut}(T M, \mathfrak{g}))$ defined by

$$
(b \cdot \Phi)\left(m^{\prime}\right)=\operatorname{Ad}_{T_{m} b} \Phi(m),
$$

where $\beta(b(m))=m^{\prime}$ defines $m \in M$, and $b \in B(G), \Phi \in B($ Aut $(T M, \mathfrak{g})), m \in M$ are arbitrary. We may therefore form the semidirect product of groups,

$$
B(G) \times \text { Ad } B(\operatorname{Aut}(T M, \mathfrak{g}))
$$

which has multiplication defined by

$$
\left(b_{1}, \Phi_{1}\right)\left(b_{2}, \Phi_{2}\right)=\left(b_{1} b_{2}, \Phi_{1} b_{1} \cdot \Phi_{2}\right) .
$$

According to Theorem 3.3(2) below, this semidirect product is isomorphic to $B\left(J^{1} G\right)$.

Secondly, suppose $G$ admits a Cartan connection $S: G \rightarrow J^{1} G$. Then $G$ acts on $\operatorname{Aut}(T M, \mathfrak{g})$ according to $g \cdot \phi:=S(g) \cdot \phi=\operatorname{Ad}_{S(g)} \phi$. With this action in hand, the pullback $G \times{ }_{\beta} \operatorname{Aut}(T M, \mathfrak{g})$ of the fibre-bundle Aut $(T M, \mathfrak{g}) \rightarrow M$ under the target projection $\beta: G \rightarrow M$ becomes a semidirect product of Lie groupoids: The source and target projections are respectively $(g, \phi) \mapsto \alpha(g)$ and $(g, \phi) \mapsto \beta(g)$, where $\alpha, \beta: G \rightarrow M$ are the corresponding projections for $G$; multiplication is defined by

$$
\left(g_{1}, \phi_{1}\right)\left(g_{2}, \phi_{2}\right):=\left(g_{1} g_{2}, \phi_{1} \operatorname{Ad}_{S\left(g_{1}\right)} \phi_{2}\right) \text {. }
$$

According to Theorem 3.3(3) below, $J^{1} G \cong G \times{ }_{\beta} \operatorname{Aut}(T M, \mathfrak{g})$ as Lie groupoids.

Theorem 3.3. For an arbitrary Lie groupoid $G$ :

(1) The map $\phi \mapsto \phi^{\vee}: \operatorname{Aut}(T M, \mathfrak{g}) \rightarrow J^{1} G$, defined by $\phi^{\vee} v=v-\phi v$, is a Lie groupoid morphism and embedding, whose image is the kernel of the natural projection $J^{1} G \rightarrow G$. This map is equivariant with respect to the natural representations of both groupoids on TM, and on $\mathfrak{g}$ :

$$
\operatorname{Ad}_{\phi} v=\phi^{T M} v, \quad \operatorname{Ad}_{\phi^{\vee}} X=\phi^{\mathfrak{g}} X, \quad v \in T M, \quad X \in \mathfrak{g} .
$$

(2) The map $a: B(G) \times$ Ad $B(\operatorname{Aut}(T M, \mathfrak{g})) \rightarrow B\left(J^{1} G\right)$ defined by

$$
a(b, \Phi)(m)=\left(\Phi\left(m^{\prime}\right)\right)^{\vee} T_{m} b, \quad m^{\prime}=\beta(b(m)),
$$

is an isomorphism of Lie groups.

Here $T_{m} b: T_{m} M \rightarrow T_{b(m)} G$ is the tangent map of $b$ at $m$, viewed as an element of $J^{1} G$, and $\beta: G \rightarrow M$ is the target projection. Also, $\left(\Phi\left(m^{\prime}\right)\right)^{\vee} T_{m} b$ denotes the product of $\left(\Phi\left(m^{\prime}\right)\right)^{\vee}$ and $T_{m} b$ in the Lie groupoid $J^{1} G$.

(3) If $S: G \rightarrow J^{1} G$ is a Cartan connection on $G$, then the map c: $G \times{ }_{\beta} \operatorname{Aut}(T M, \mathfrak{g}) \rightarrow J^{1} G$ defined by $c(g, \phi)=\phi^{\vee} S(g)$ is an isomorphism of Lie groupoids. 


\subsection{Source connections and parallel actions}

Before proving the preceding results, we introduce some terminology and notation we need when calculating the derivatives of paths in a groupoid $G$ of the form $g(t) h(t)$ (and later when we establish a geometric interpretation of an infinitesimalized Cartan connection.)

By a source connection on $G$, we shall mean a rank- $n$ subbundle $D \subset T G$ such that: (i) $T G=$ $D+\operatorname{ker} T \alpha$; and (ii) $D(m)=T_{m} M$ for all $m \in M \subset G$. Here $n:=\operatorname{dim}(M)$ and $\alpha: G \rightarrow M$ is the source projection. A Cartan connection $D$, as defined in Section 1.1 above, is a source connection. While Cartan connections need not exist in general, one can always find a source connection locally (which will suffice for present purposes) and even globally if we work in the $C^{\infty}$ category.

Suppose $a<0<b$. Fixing a source connection $D$ on $G$, and a smooth path $\gamma:(a, b) \rightarrow M$ on the base $M$, we can define a local form of parallel translation along $\gamma$. This parallel translation will be expressed in the language of Lie groupoid actions; see, e.g., [23, Section 1.6].

To simplify our description, we assume $\gamma$ is regular and simple, so that the pullback $\gamma^{*} G$ of the surjective submersion $\alpha: G \rightarrow M$ along $\gamma:(a, b) \rightarrow M$ can be identified with a submanifold of $G$, namely the pre-image of $\gamma((a, b))$ under $\alpha$. This pre-image is a union of source-fibres, $P_{t}=\alpha^{-1}(\gamma(t)), a<t<b$. For any $U \subset \gamma^{*} G$ we write $(a, b)^{2} \times_{\gamma} U$ for the set of all $\left(t_{1}, t_{2}, g\right) \in$ $(a, b)^{2} \times U$ such that $\alpha(g)=\gamma\left(t_{1}\right)$.

We claim that for any point $g_{0} \in P_{0} \subset \gamma^{*} G$ (or, more generally, any finite number of points in $\left.P_{0}\right)$, there exists - shrinking the interval $(a, b)$ if necessary - an open neighborhood $U$ of $g_{0}$ in $\gamma^{*} G$, and an action of the pair groupoid $(a, b)^{2}$ on the restriction $\alpha: U \rightarrow \gamma((a, b))$, denoted $\left(t_{1}, t_{2}, g\right) \mapsto A_{t_{1}, t_{2}}^{\gamma}(g):(a, b)^{2} \times_{\gamma} U \rightarrow U$, such that the path $t \mapsto A_{t_{0}, t}^{\gamma}(g)$ is $D$-horizontal, for any $t_{0} \in(a, b)$ and $g \in P_{t_{0}}$. Indeed, $t \mapsto A_{t_{0}, t}^{\gamma}(g)$ is then the $D$-horizontal lift through $\alpha: G \rightarrow M$, of the path $t \mapsto \gamma(t)$, that passes through $g$ at time $t_{0}$. The existence of the action follows, for example, from the observation that the connection $D$ on $\alpha: G \rightarrow M$ pulls back to a rank-one connection on the pullback $\gamma^{*} G \rightarrow(a, b)$; finding horizontal paths then amounts to integrating a vector field, to which the standard existence and uniqueness theory for flows applies.

We will call the action $A^{\gamma}$ defined above the parallel action along $\gamma$ associated with the connection $D$. Note that $A_{t_{1}, t_{2}}^{\gamma}(m)=m$ because $D(m)=T_{m} M$ for all $m \in M$.

\subsection{Multiplication in $J^{1} G$ in special cases}

The technical part of the proof of Theorem 3.3 is to derive product formulas in a few special cases. Referring to Fig. 1 below, we have:

Lemma 3.4. Suppose $g \in G$ begins at $m \in M$ and ends at $m^{\prime} \in M$. Then, for all $\mu, \nu \in J_{g}^{1} G$ and $\left.\phi \in \operatorname{Aut}(T M, \mathfrak{g})\right|_{m}$ :

(1) $\mu \phi^{\vee}(v)=\mu\left(\phi^{T M} v\right)+T L_{g} \cdot T I \cdot(\phi v)$, where $v \in T_{m} M$ is arbitrary;

(2) $\nu \mu^{-1}=\psi^{\vee}$, where

$$
\psi v=T R_{g^{-1}} \cdot\left(\mu\left(\operatorname{Ad}_{\mu}^{-1} v\right)-\nu\left(\operatorname{Ad}_{\mu}^{-1} v\right)\right), \quad v \in T_{m^{\prime}} M
$$

(3) $\mu \phi^{\vee} \mu^{-1}=\left(\operatorname{Ad}_{\mu} \phi\right)^{\vee}$.

Also, if $\mu, \nu$ and $\phi$ above are related by $\nu=\mu \phi^{\vee}$, then:

(4) $\mu(v)-\nu(v)=T R_{g} \cdot \operatorname{Ad}_{\mu}(\phi v), v \in T_{m} M$.

Here $T I, T L_{g}, T R_{g^{-1}}: T G \rightarrow T G$ denote the tangent maps for inversion, left-multiplication by $g$, and right-multiplication by $g^{-1}$. The map $\phi \mapsto \phi^{\vee}$ was defined in Theorem 3.3(1). 

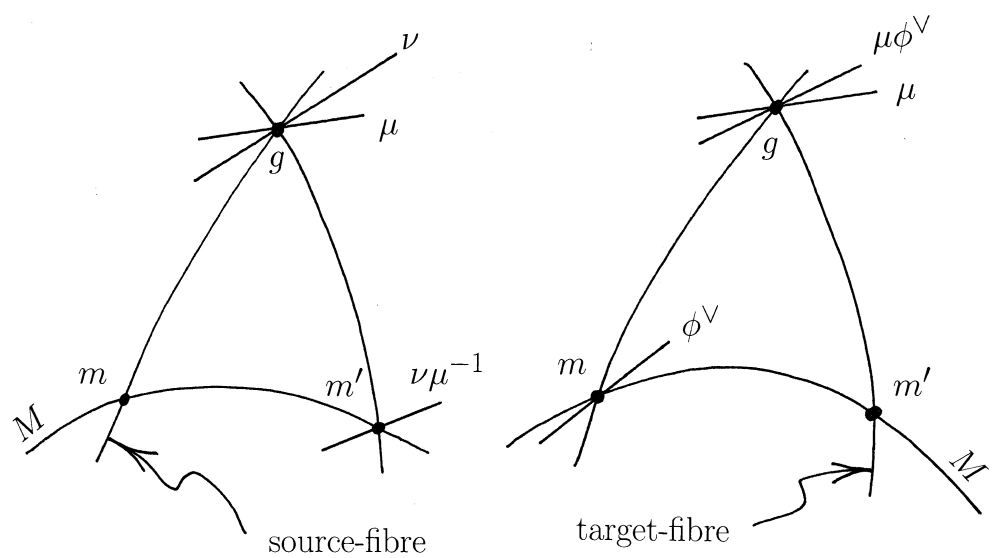

Figure 1. Schematic showing the first two products considered in Lemma 3.4. In the lemma we view elements $\mu, \nu, \phi^{\vee} \in J^{1} G$ as linear maps but in the figure they are represented by their images (straight-line segments).

Proof. We first prove (1). Let $b_{1}$ and $b_{2}$ be local bisections of $G$ (viewed as local right inverses of the source-projection $G \rightarrow M$ ) such that

$$
\mu=T_{m} b_{1}, \quad \phi^{\vee}=T_{m} b_{2} .
$$

Let $v \in T_{m} M$ be arbitrary and $\gamma^{\prime}:(-\epsilon, \epsilon) \rightarrow M$ be a simple regular path with $\gamma^{\prime}(0)=m^{\prime}$ and $\dot{\gamma}^{\prime}(0)=v$, where a dot denotes derivative. According to the definition of multiplication in $J^{1} G$, we have

$$
\mu \phi^{\vee}(v)=\left.\frac{d}{d t} g(t) h(t)\right|_{t=0},
$$

where

$$
g(t):=b_{1}(\gamma(t)), \quad h(t):=b_{2}\left(\gamma^{\prime}(t)\right), \quad \gamma(t):=\beta(h(t))=\beta\left(b_{2}\left(\gamma^{\prime}(t)\right)\right) .
$$

Here $\beta: G \rightarrow M$ denotes the target projection. We note in passing that

$$
\dot{\gamma}(0)=\phi^{T M} v .
$$

Unfortunately, we cannot apply the chain rule directly to (3.5) because $g(s) h(t)$ need not be defined unless $s=t$. To overcome this difficulty, equip $G$ with an source connection $D$, as described in Section 3.6 above, and let

$$
\left(t_{1}, t_{2}, g\right) \mapsto A_{t_{1}, t_{2}}^{\gamma}(g):(-\epsilon, \epsilon)^{2} \times_{\gamma} U \rightarrow U
$$

denote the associated parallel action along $\gamma$. Here $\epsilon>0$, and we may arrange $U \subset G$ to be an open set containing both elements $m, g \in G$. We may now write

$$
g(t) h(t)=A_{0, t}^{\gamma}(\tilde{g}(t))\left(A_{0, t}^{\gamma}\left(\tilde{h}(t)^{-1}\right)\right)^{-1}
$$

where

$$
\begin{aligned}
& \tilde{g}(t):=A_{t, 0}^{\gamma}(g(t)), \\
& \tilde{h}(t):=\left(A_{t, 0}^{\gamma}\left(h(t)^{-1}\right)\right)^{-1} .
\end{aligned}
$$


Noting that $g(t) h(t)=f(t, t)$, where

$$
f(s, t)=A_{0, s}^{\gamma}(\tilde{g}(t))\left(A_{0, s}^{\gamma}\left(\tilde{h}(t)^{-1}\right)\right)^{-1}
$$

is well-defined for all $(s, t) \in \mathbb{R}^{2}$ sufficiently close to the origin, we may now apply the chain rule to (3.5):

$$
\begin{aligned}
\phi^{\vee} \mu(v) & =\left.\frac{d}{d s} f(s, 0)\right|_{s=0}+\left.\frac{d}{d t} f(0, t)\right|_{t=0} \\
& =\left.\frac{d}{d s} A_{0, s}^{\gamma}(\tilde{g}(0))\left(A_{0, s}^{\gamma}\left(\tilde{h}(0)^{-1}\right)\right)^{-1}\right|_{s=0}+\left.\frac{d}{d t} A_{0,0}^{\gamma}(\tilde{g}(t))\left(A_{0,0}^{\gamma}\left(\tilde{h}(t)^{-1}\right)\right)^{-1}\right|_{t=0} \\
& =w+\left.\frac{d}{d t} \tilde{g}(t) \tilde{h}(t)\right|_{t=0},
\end{aligned}
$$

where $w$ is the $D$-horizontal lift of $\dot{\gamma}(0)=\phi^{T M} v$ to an element of $D(g) \subset T_{g} G$.

Noting that $\tilde{g}(s) \tilde{h}(t)$ is defined for all $(s, t) \in \mathbb{R}^{2}$ sufficiently close to the origin, we may apply the chain rule directly to obtain

$$
\left.\frac{d}{d t} \tilde{g}(t) \tilde{h}(t)\right|_{t=0}=\dot{\tilde{g}}(0)+T L_{g} \dot{\tilde{h}}(0) .
$$

It remains to compute $\dot{\tilde{g}}(0)$ and $\dot{\tilde{h}}(0)$. From (3.8) we have

$$
g(t)=A_{0, t}^{\gamma}(\tilde{g}(t))=\left.A_{0, s}^{\gamma}(\tilde{g}(t))\right|_{s=t},
$$

so that the chain rule gives

$$
\dot{g}(0)=\left.\frac{d}{d s} A_{0, s}^{\gamma}(\tilde{g}(0))\right|_{s=0}+\left.\frac{d}{d t} A_{0,0}^{\gamma}(\tilde{g}(t))\right|_{t=0}=w+\dot{\tilde{g}}(0) .
$$

Therefore

$$
\dot{\tilde{g}}(0)=\dot{g}(0)-w .
$$

Similar arguments applied to (3.9) give us

$$
\dot{\tilde{h}}(0)=\dot{h}(0)-\phi^{T M} v .
$$

These last two results and (3.11) imply

$$
\left.\frac{d}{d t} \tilde{g}(t) \tilde{h}(t)\right|_{t=0}=\dot{g}(0)-w+T L_{g} \cdot\left(\dot{h}(0)-\phi^{T M} v\right) .
$$

Equation (3.10) now reads

$$
\phi^{\vee} \mu(v)=\dot{g}(0)+T L_{g} \cdot\left(\dot{h}(0)-\phi^{T M} v\right) .
$$

Finally, appealing to (3.6), (3.7) and (3.4), we obtain

$$
\phi^{\vee} \mu(v)=\mu\left(\phi^{T M} v\right)+T L_{g} \cdot\left(\phi^{\vee}(v)-\phi^{T M} v\right) .
$$

Noting the identity $\# X-X=T I \cdot X, X \in \mathfrak{g}$, one obtains

$$
T L_{g} \cdot\left(\phi^{\vee}(v)-\phi^{T M} v\right)=T L_{g} \cdot(\# \phi v-\phi v)=T L_{g} \cdot T I \cdot(\phi v) .
$$

Formula (1) now follows. 
The strategy for proving (2) is very similar and is omitted. Regarding (3), we have, putting $\nu=\mu \phi^{\vee}$ in $(2), \mu \phi^{\vee} \mu^{-1}=\psi^{\vee}$, where

$$
\psi v=T R_{g^{-1}} \cdot\left(\mu(w)-\left(\mu \phi^{\vee}\right)(w)\right),
$$

and $w=\operatorname{Ad}_{\mu}^{-1} v$. Substituting (1):

$$
\begin{aligned}
\psi v & =T R_{g^{-1}} \cdot\left(\mu(w)-\mu\left(\phi^{T M} w\right)-T L_{g} \cdot T I \cdot(\phi w)\right) \\
& =T R_{g^{-1}} \cdot\left(\mu(\# \phi w)-T L_{g} \cdot T I \cdot(\phi w)\right) \\
& =\operatorname{Ad}_{\mu}(\phi w)=\operatorname{Ad}_{\mu}\left(\phi \operatorname{Ad}_{\mu}^{-1} v\right)=\left(\operatorname{Ad}_{\mu} \phi\right) v .
\end{aligned}
$$

Finally, we note that (4) is a straightforward consequence of (3) and (2).

\subsection{Proof of Theorem 3.3}

That the map $\phi \mapsto \phi^{\vee}$ in Theorem 3.3(1) is an embedding, with image the kernel of $J^{1} G \rightarrow G$, is readily checked, as are the equivariance claims. To prove $\psi^{\vee} \phi^{\vee}=(\psi \phi)^{\vee}$, take $g=m$ and $\mu=\psi^{\vee}$ in Lemma 3.4(1) and apply the identity $T I \cdot X=\# X-X$, for $X \in \mathfrak{g}$. Note that multiplication in $\operatorname{Aut}(T M, \mathfrak{g})$ was defined in (3.3).

To prove Theorem 3.3(2), let $b_{1}, b_{2}$ be bisections of $G$ and $\Phi_{1}, \Phi_{2}$ sections of $\operatorname{Aut}(T M, \mathfrak{g})$. Let $m \in M$ be given and define $m^{\prime}=\beta\left(b_{2}(m)\right)$ and $m^{\prime \prime}=\beta\left(b_{1}\left(m^{\prime}\right)\right)$. We compute

$$
\begin{aligned}
a\left(\left(b_{1}, \Phi_{1}\right)\left(b_{2}, \Phi_{2}\right)\right)(m) & =a\left(b_{1} b_{2}, \Phi_{1} b_{1} \cdot \Phi_{2}\right)(m)=\left(\left(\Phi_{1} b_{1} \cdot \Phi_{2}\right)\left(m^{\prime \prime}\right)\right)^{\vee} T_{m}\left(b_{1} b_{2}\right) \\
& =\left(\Phi_{1}\left(m^{\prime \prime}\right) \operatorname{Ad}_{T_{m^{\prime}} b_{1}} \Phi_{2}\left(m^{\prime}\right)\right)^{\vee} T_{m}\left(b_{1} b_{2}\right) \\
& \text { by Theorem } 3.3(1)\left(\Phi_{1}\left(m^{\prime \prime}\right)\right)^{\vee}\left(\operatorname{Ad}_{T_{m^{\prime}} b_{1}} \Phi_{2}\left(m^{\prime}\right)\right)^{\vee} T_{m}\left(b_{1} b_{2}\right) \\
& \text { by Lemma } 3.4(3) \\
= & \left(\Phi_{1}\left(m^{\prime \prime}\right)\right)^{\vee} T_{m^{\prime}} b_{1}\left(\Phi_{2}\left(m^{\prime}\right)\right)^{\vee}\left(T_{m^{\prime}} b_{1}\right)^{-1} T_{m}\left(b_{1} b_{2}\right) \\
& =\left(\Phi_{1}\left(m^{\prime \prime}\right)\right)^{\vee} T_{m^{\prime}} b_{1}\left(\Phi_{2}\left(m^{\prime}\right)\right)^{\vee} T_{m} b_{2}=a\left(b_{1}, \Phi_{1}\right) a\left(b_{2}, \Phi_{2}\right) .
\end{aligned}
$$

Regarding, Theorem 3.3(3), the reader will be readily convinced that the map

$$
c: G \times_{\beta} \operatorname{Aut}(T M, \mathfrak{g}) \rightarrow J^{1} G
$$

defined by $c(g, \phi)=\phi^{\vee} S(g)$ is a diffeomorphism. To show that it is a morphism of groupoids, let $\left(g_{1}, \phi_{1}\right),\left(g_{2}, \phi_{2}\right) \in G \times_{\beta} \operatorname{Aut}(T M, \mathfrak{g})$ be given. Then

$$
\begin{aligned}
& c\left(g_{1}, \phi_{1}\right) c\left(g_{2}, \phi_{2}\right)=\phi_{1}^{\vee} S\left(g_{1}\right) \phi_{2}^{\vee} S\left(g_{2}\right)=\phi_{1}^{\vee} S\left(g_{1}\right) \phi_{2}^{\vee} S\left(g_{1}\right)^{-1} S\left(g_{1} g_{2}\right) \\
& \text { by Lemma } 3.4(3) \phi_{1}^{\vee}\left(\operatorname{Ad}_{S\left(g_{1}\right)} \phi_{2}\right)^{\vee} S\left(g_{1} g_{2}\right) \\
& \text { by Theorem } \stackrel{3.3(1)}{=}\left(\phi_{1} \operatorname{Ad}_{S\left(g_{1}\right)} \phi_{2}\right)^{\vee} S\left(g_{1} g_{2}\right) \\
& =c\left(g_{1} g_{2}, \phi_{1} \operatorname{Ad}_{S\left(g_{1}\right)} \phi_{2}\right)=c\left(\left(g_{1}, \phi_{1}\right)\left(g_{2}, \phi_{2}\right)\right) \text {. }
\end{aligned}
$$

\section{On the infinitesimalization of Cartan connections}

Let $D \subset T G$ be a Cartan connection on a Lie groupoid $G$ over $M$. Then, in the terminology of Section 3.6, $D$ is also a globally defined source-connection on $G$. We differentiate the corresponding parallel action of $D$, along curves in $M$, to obtain a linear connection $\nabla^{D}$ on the Lie algebroid $\mathfrak{g}$ of $G$. Theorem 4.1 below states that this 'geometrically' defined connection $\nabla^{D}$ coincides with the infinitesimal Cartan connection $\nabla$, defined 'algebraically' in Section 1.3. 


\subsection{Differentiating the parallel action defined by a source connection}

Let $D$ be an arbitrary source connection on $G$. Let $v \in T_{m} M$ be an arbitrary tangent vector at some point $m \in M$, and $\gamma:(-\epsilon, \epsilon) \rightarrow M$ a simple regular path with $\gamma(0)=m$ and $\dot{\gamma}(0)=v$, where a dot denotes derivative. Shrinking $\epsilon$ if necessary, we have, for some open neighborhood $U$ of $m$ in $\gamma^{*} G$, the corresponding parallel action $A^{\gamma}:(-\epsilon, \epsilon)^{2} \times_{\gamma} U \rightarrow U$ defined in Section 3.6 - an action of the pair groupoid $(-\epsilon, \epsilon)^{2}$ on $U$. We obtain an action $a^{\gamma}$ of $(-\epsilon, \epsilon)^{2}$ on the pullback $\gamma^{*} \mathfrak{g}-$ which, for simplicity, we identify with a submanifold of $\mathfrak{g}$ - by differentiating:

$$
a_{t_{1}, t_{2}}^{\gamma}\left(\left.\frac{d}{d s} g(s)\right|_{s=0}\right):=\left.\frac{d}{d s} A_{t_{1}, t_{2}}^{\gamma} g(s)\right|_{s=0} .
$$

Here we view an element of $\left.\left.\gamma^{*} \mathfrak{g}\right|_{m} \cong \mathfrak{g}\right|_{m}$ as the derivative of some path $s \mapsto g(s) \in G$ lying in a source-fibre of $G$. A linear connection $\nabla^{D}$ on $\mathfrak{g}$ is now defined by

$$
\nabla_{v}^{D} X:=\left.\partial_{t} a_{t, 0}^{\gamma} X(\gamma(t))\right|_{t=0}, \quad X \in \Gamma(\mathfrak{g}) .
$$

Notation. Here and in the sequel $\partial_{t}$ indicates a derivative with respect to $t$ of a vector-valued function of $t$, identified with an element of the underlying vector space and not a tangent vector (i.e., with base-point 'forgotten').

Here is the main result of the present section:

Theorem 4.1. If $D \subset T G$ is a Cartan connection on $G$ then $\nabla^{D}$ coincides with the corresponding infinitesimal Cartan connection $\nabla$ defined in Section 1.3.

The proof of this theorem is given in Section 4.3 after necessary preparations.

\subsection{The Lie algebroid of $\operatorname{Aut}(T M, \mathfrak{g})$}

Implicit in the discussion at the beginning of Section 3 is an identification of the Lie algebroid of our model Aut $(T M, \mathfrak{g})$ of the kernel of $J^{1} G \rightarrow G$ with the kernel $T^{*} M \otimes \mathfrak{g}$ of $J^{1} \mathfrak{g} \rightarrow \mathfrak{g}$. To prove the preceding theorem we must make this identification explicit. To this end, let $\mathcal{L}(G)$ denote the (abstract) Lie algebroid of any Lie groupoid $G$. Then an element of $\mathcal{L}(\operatorname{Aut}(T M, \mathfrak{g}))$ is of the form $\left.\frac{d}{d t} \phi_{t}\right|_{t=0}$, for some path $t \mapsto \phi_{t} \in \operatorname{Aut}(T M, \mathfrak{g})$ lying completely in some source-fibre (= target-fibre) of $\operatorname{Aut}(T M, \mathfrak{g})$; the latter is simply an open neighborhood of zero in a vector space

$$
\left.\left(T^{*} M \otimes \mathfrak{g}\right)\right|_{m}, \quad m \in M .
$$

Of course, we also require that $\phi_{0}$ be an identity element of $\operatorname{Aut}(T M, \mathfrak{g})$, which just means that $\phi_{0}$ is the zero element of $\left.\left(T^{*} M \otimes \mathfrak{g}\right)\right|_{m}$. There is a natural isomorphism of vector bundles $\theta^{\prime}: \mathcal{L}(\operatorname{Aut}(T M, \mathfrak{g})) \rightarrow T^{*} M \otimes \mathfrak{g}$ defined by

$$
\theta^{\prime}\left(\left.\frac{d}{d t} \phi_{t}\right|_{t=0}\right) v=\left.\partial_{t} \phi_{t} v\right|_{t=0}
$$

This isomorphism is the appropriate one for present purposes, by virtue of the following:

Proposition 4.2. Defining $\theta^{\prime}$ as above, we have the following commutative diagram with exact rows:

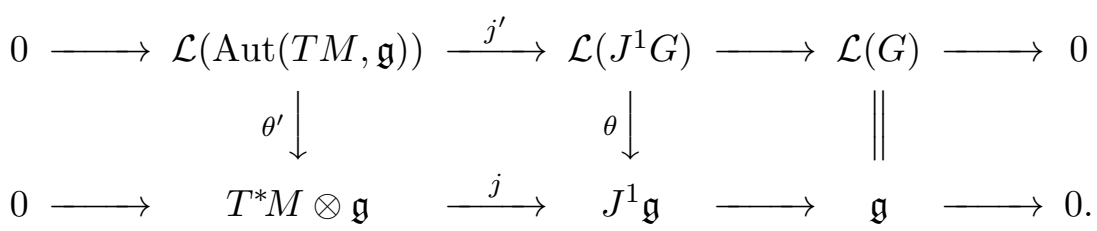


Here $\theta$ denotes the isomorphism described in Section $3.1 ; j^{\prime}$ denotes the derivative of the map $\phi \mapsto \phi^{\vee}: \operatorname{Aut}(T M, \mathfrak{g}) \rightarrow J^{1} G$ defined in Theorem 3.3(1); $j$ denotes the map which induces a corresponding map of section spaces given by $d f \otimes X \mapsto f J^{1} X-J^{1}(f X)$.

Key in the proof of the proposition is the following technical result applied again in Section 4.3 below:

Lemma 4.3. Let $\alpha: G \rightarrow M$ denote the source map of $G$ and let $D$ be any source-connection. For each $g \in G$ beginning at some $m=\alpha(g)$, let $S(g): T_{m} M \rightarrow D(g)$ denote the inverse of the restriction $T_{g} \alpha: D(g) \rightarrow T_{m} M$ of the tangent map T $\alpha$. (In other words, $S(g)(v)$ is the $D$-horizontal lift of $v \in T_{m} M$ to a vector at $g$; if $D$ is a Cartan connection, we view $S(g) \in J^{1} G$ and $S: G \rightarrow J^{1} G$ has the meaning in Section 1.1.) Then, for any function $f$ on $G$ defined in a neighborhood of $m$, one has

$$
\left\langle d f, \nabla_{v}^{D} X\right\rangle=\left.\partial_{t}\left\langle d f, T_{m}\left(\Phi_{X^{\mathrm{R}}}^{t} \circ \iota_{M}\right) \cdot v\right\rangle\right|_{t=0}-\left.\partial_{t}\left\langle d f, S\left(\Phi_{X^{\mathrm{R}}}^{t}(m)\right)(v)\right\rangle\right|_{t=0} .
$$

Proof. Adopting the notation of Section 4.1 with $v=\dot{\gamma}(0)$, we calculate,

$$
\begin{aligned}
\left\langle d f, \nabla_{v}^{D} X\right\rangle & =\left.\partial_{s}\left\langle d f, a_{s, 0}^{\gamma} X(\gamma(s))\right\rangle\right|_{s=0}=\left.\partial_{s} \partial_{t} f\left(A_{s, 0}^{\gamma} \Phi_{X^{\mathrm{R}}}^{t}(\gamma(s))\right)\right|_{t=0, s=0} \\
& =\left.\partial_{t} \partial_{s} f(g(s, t))\right|_{t=0, s=0},
\end{aligned}
$$

i.e.,

$$
\left\langle d f, \nabla_{v}^{D} X\right\rangle=\left.\partial_{t}\left\langle d f,\left.\frac{\partial}{\partial s} g(s, t)\right|_{s=0}\right\rangle\right|_{t=0},
$$

where $g(s, t)=A_{s, 0}^{\gamma} \Phi_{X^{\mathrm{R}}}^{t}(\gamma(s))$ is defined for all $(s, t)$ sufficiently close to $(0,0)$, and we have appealed to the equality of mixed partial derivatives. Now we can write $\Phi_{X^{\mathrm{R}}}^{t}(\gamma(s))=A_{0, s}^{\gamma} g(s, t)$ and notice that $A_{0, s_{1}}^{\gamma} g\left(s_{2}, t\right)$ is defined for all $\left(s_{1}, s_{2}, t\right)$ sufficiently close to $(0,0,0)$. It follows that we may differentiate with respect to $s$ at $s=0$ and apply the chain rule:

$$
\left.\frac{\partial}{\partial s} \Phi_{X \mathrm{R}}^{t}(\gamma(s))\right|_{s=0}=\left.\frac{\partial}{\partial s} A_{0, s}^{\gamma} g(0, t)\right|_{s=0}+\left.\frac{\partial}{\partial s} A_{0,0}^{\gamma} g(s, t)\right|_{s=0},
$$

i.e.,

$$
T_{m}\left(\Phi_{X^{\mathrm{R}}}^{t} \circ \iota_{M}\right) \cdot v=S\left(\Phi_{X^{\mathrm{R}}}^{t}\right)(v)+\left.\frac{\partial}{\partial s} g(s, t)\right|_{s=0} .
$$

We conclude that

$$
\left.\frac{\partial}{\partial s} g(s, t)\right|_{s=0}=T_{m}\left(\Phi_{X^{\mathrm{R}}}^{t} \circ \iota_{M}\right) \cdot v-S\left(\Phi_{X^{\mathrm{R}}}^{t}(m)\right)(v) .
$$

Substituting this into (4.1) gives the desired result.

Proof of Proposition 4.2. Exactness of the top row follows from Theorem 3.3(1); exactness of the bottom row is immediate. It remains to show that for any $\zeta \in \mathcal{L}(\operatorname{Aut}(T M, \mathfrak{g}))$, we have

$$
\theta\left(j^{\prime}(\zeta)\right)=j\left(\theta^{\prime}(\zeta)\right)
$$

By exactness, and the fact that $\theta$ is an isomorphism, the element $j^{\prime}(\zeta)$ is in the image of the composite morphism

$$
T^{*} M \otimes \mathfrak{g} \stackrel{j}{\rightarrow} J^{1} \mathfrak{g} \stackrel{\theta^{-1}}{\longrightarrow} \mathcal{L}\left(J^{1} G\right) .
$$


But any element of $T^{*} M \otimes \mathfrak{g}$ is of the form $-\nabla X(m)$, where $\nabla$ is an arbitrary linear connection on $\mathfrak{g}, m \in M$, and $X$ is a local section of $\mathfrak{g}$ with $X(m)=0$. Since in that case $j(-\nabla X(m))=$ $J_{m}^{1} X$, it follows, from formula (3.2) for $\theta^{-1}$ that

$$
j^{\prime}(\zeta)=\left.\frac{d}{d t} T_{m}\left(\Phi_{X \mathrm{R}}^{t} \circ \iota_{M}\right)\right|_{t=0},
$$

for some $m \in M$ and $X \in \Gamma(\mathfrak{g})$ with $X(m)=0$. Furthermore, we have, by construction, $\theta\left(j^{\prime}(\zeta)\right)=j(-\nabla X(m))$. Consequently, to prove (4.2), it suffices, since $j$ is injective, to show that

$$
\nabla X(m)=-\theta^{\prime}(\zeta)
$$

Now the map $j^{\prime}$ is the derivative of the embedding $\phi \mapsto \phi^{\vee}: \operatorname{Aut}(T M, \mathfrak{g}) \rightarrow J^{1} G$ defined in Theorem 3.3(1). In view of this fact and (4.3), there is a path $t \mapsto \phi_{t} \operatorname{in} \operatorname{Aut}(T M, \mathfrak{g})$ such that $\phi_{t}^{\vee}=T_{m}\left(\Phi_{X^{\mathrm{R}}}^{t} \circ \iota_{M}\right)$ and $\zeta=\left.\frac{d}{d t} \phi_{t}\right|_{t=0}$. To prove (4.4) we will apply both sides to some tangent vector $v=\dot{\gamma}(0) \in T_{m} M$ and test the resulting elements of $\left.\mathfrak{g}\right|_{m}$ on a test function $f$, defined on $G$, in some neighborhood of $m$. It will be convenient to take $\nabla=\nabla^{D}$, where $D \subset T G$ is an arbitrary source connection on $G$. Then, applying the preceding lemma, and using the fact that $\Phi_{X^{\mathrm{R}}}^{t}(m)=m($ since $X(m)=0)$ we compute

$$
\begin{aligned}
\left\langle d f, \nabla_{v} X\right\rangle=\left\langle d f, \nabla_{v}^{D} X\right\rangle & =\left.\partial_{t}\left\langle d f, T_{m}\left(\Phi_{X^{\mathrm{R}}}^{t} \circ \iota_{M}\right) \cdot v\right\rangle\right|_{t=0}-\left.\partial_{t}\langle d f, v\rangle\right|_{t=0} \\
& =\left.\partial_{t}\left\langle d f, \phi_{t}^{\vee}(v)-v\right\rangle\right|_{t=0}=\left.\partial_{t}\left\langle d f,-\phi_{t} v\right\rangle\right|_{t=0} \\
& =-\left.\left\langle d f, \partial_{t}\left(\phi_{t} v\right)\right\rangle\right|_{t=0}=-\left\langle d f, \theta^{\prime}\left(\left.\frac{d}{d t} \phi_{t}\right|_{t=0}\right) v\right\rangle \\
& =-\left\langle d f, \theta^{\prime}(\zeta) v\right\rangle
\end{aligned}
$$

which proves (4.4).

\subsection{The proof of Theorem 4.1}

Lemma 4.4. Let $g_{t}$ be a path in $G$ such that $g_{0}=m \in M$ and $g_{t}$ begins at $m$ for all $t$. Suppose $\mu_{t}$ and $\nu_{t}$ are two paths in $J^{1} G$ with common projection $g_{t}$ onto $G$, and such that $\mu_{0}$ and $\nu_{0}$ coincide with the identity element $T_{m} \iota_{M}$ of $J^{1} G$ (see Fig. 2). Then there exists a (necessarily unique) $\psi \in T^{*} M \otimes \mathfrak{g}$ such that:

(1) $\theta\left(\left.\frac{d}{d t} \mu_{t}\right|_{t=0}-\left.\frac{d}{d t} \nu_{t}\right|_{t=0}\right)=j(\psi)$, and

(2) $\langle d f, \psi v\rangle=-\left.\partial_{t}\left\langle d f, \mu_{t}(v)\right\rangle\right|_{t=0}+\left.\partial_{t}\left\langle d f, \nu_{t}(v)\right\rangle\right|_{t=0}$,

for all functions $f$ on $G$ defined in some neighborhood of $m$, and all $v \in T_{m} M$.

For the definition of $\theta$ and $j$ see Proposition 4.2.

Proof of Lemma 4.4. Since $\mu_{t}$ and $\nu_{t}$ have common projection onto $G$, we have $\nu_{t}=\phi_{t}^{\vee} \mu_{t}$ (multiplication in $J^{1} G$ ) for some $\phi_{t} \in T^{*} M \otimes \mathfrak{g}$. We have $\phi_{0}^{\vee}=T_{m} \iota_{M}$, i.e., $\phi_{0}=0$. Differentiating and applying the chain rule, we obtain

$$
\left.\frac{d}{d t} \mu_{t}\right|_{t=0}-\left.\frac{d}{d t} \nu_{t}\right|_{t=0}=-\left.\frac{d}{d t} \phi_{t}^{\vee}\right|_{t=0}=-j^{\prime}\left(\left.\frac{d}{d t} \phi_{t}\right|_{t=0}\right)
$$

where $j^{\prime}$ is defined in Proposition 4.2. Applying $\theta$ to both sides, and using commutativity of the diagram appearing in that proposition, we obtain (1) if we take $\psi=-\theta^{\prime}\left(\frac{d}{d t} \phi_{t}\right)$. In that case, by the definition of $\theta^{\prime}$, we have

$$
\psi v=-\left.\partial_{t}\left(\phi_{t} v\right)\right|_{t=0}, \quad v \in T_{m} M .
$$




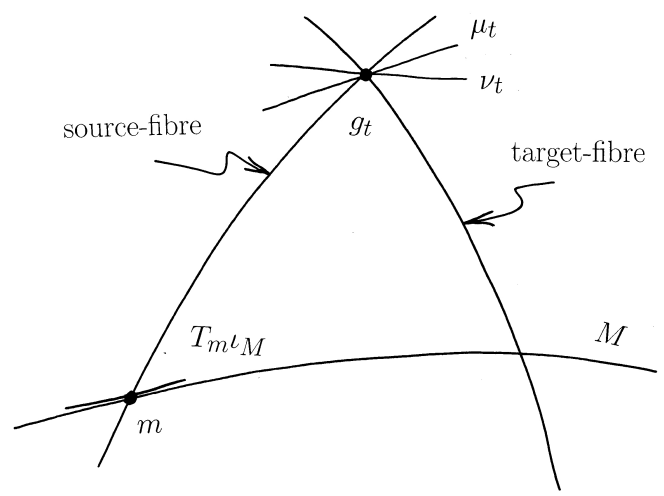

Figure 2. Schematic showing the paths in $J^{1} G$ considered in Lemma 4.4.

On the other hand, applying Lemma 3.4(4), we have

$$
T R_{g_{t}} \cdot \operatorname{Ad}_{\mu_{t}}\left(\phi_{t} v\right)=\mu_{t}(v)-\nu_{t}(v) .
$$

Applying $d f$ to both sides and differentiating using the chain rule on the left, we obtain

$$
\left.\partial_{t}\left\langle d f, T R_{g_{t}} \cdot \operatorname{Ad}_{\mu_{t}} \zeta_{T G}(m)\right\rangle\right|_{t=0}+\left.\partial_{t}\left\langle d f, \phi_{t} v\right\rangle\right|_{t=0}=\left.\partial_{t}\left\langle d f, \mu_{t}(v)\right\rangle\right|_{t=0}-\left.\partial_{t}\left\langle d f, \nu_{t}(v)\right\rangle\right|_{t=0} .
$$

Here $\zeta_{T G}$ denotes the zero-section of $T G$. We have $T R_{g_{t}} \cdot \operatorname{Ad}_{\mu_{t}} \zeta_{T G}(m)=\zeta_{T G}\left(g_{t}\right)$, which means the first term on the left vanishes, giving us

$$
\left\langle d f,\left.\partial_{t}\left(\phi_{t} v\right)\right|_{t=0}\right\rangle=\left.\partial_{t}\left\langle d f, \mu_{t}(v)\right\rangle\right|_{t=0}-\left.\partial_{t}\left\langle d f, \nu_{t}(v)\right\rangle\right|_{t=0}
$$

This, together with (4.5), establishes (2).

Now suppose $D \subset T G$ is a Cartan connection on $G$, let $S: G \rightarrow J^{1} G$ denote the corresponding morphism of Lie groupoids, and $\nabla$ the corresponding infinitesimal Cartan connection on $\mathfrak{g}=\mathcal{L}(G)$. We are now in a position to prove $\nabla^{D}=\nabla$.

First, let us make the 'algebraic' definition of $\nabla$ in Section 1.3 more explicit. If we denote the (abstract) derivative of $S: G \rightarrow J^{1} G$ by $d S: \mathcal{L}(G) \rightarrow \mathcal{L}\left(J^{1} G\right)$ then we obtain a splitting $s: \mathfrak{g} \rightarrow J^{1} \mathfrak{g}$ of the exact sequence appearing in the bottom row of the commutative diagram in Proposition 4.2 , by putting $s=\theta \circ d S$. Then, $\nabla$ is defined implicitly by

$$
j(\nabla X(m))=-J_{m}^{1} X+s(X(m)) .
$$

Recalling the definition of $\theta$ given in (3.2), we may rewrite this as

$$
j(\nabla X(m))=\theta\left(-\left.\frac{d}{d t} T_{m}\left(\Phi_{X^{\mathrm{R}}}^{t} \circ \iota_{M}\right)\right|_{t=0}+\left.\frac{d}{d t} S\left(\Phi_{X^{\mathrm{R}}}^{t}(m)\right)\right|_{t=0}\right) .
$$

Applying the preceding lemma, we obtain

$$
\left\langle d f, \nabla_{v} X\right\rangle=\left.\partial_{t}\left\langle d f, T_{m}\left(\Phi_{X^{\mathrm{R}}}^{t} \circ \iota_{M}\right) \cdot v\right\rangle\right|_{t=0}-\left.\partial_{t}\left\langle d f, S\left(\Phi_{X^{\mathrm{R}}}^{t}(m)\right)(v)\right\rangle\right|_{t=0} .
$$

Here $v \in T_{m} M$ is arbitrary, as is the locally defined function $f$ on $G$. Our claim $\nabla=\nabla^{D}$ now follows from Lemma 4.3.

\section{Proof of Theorem 1.2}

In this section $D$ is a Cartan connection on $G, S: G \rightarrow J^{1} G$ the corresponding right-inverse for the natural projection $J^{1} G \rightarrow G$, and $\nabla$ the corresponding infinitesimal Cartan connection. 


\subsection{Proof of Theorem 1.2(1)}

Let $\mathcal{F}$ be the foliation on $V \subset G$ integrating $D$. For each $m \in M$ let $P_{m} \subset V$ be the intersection of $V$ with the source fibre of $G$ over $m$. Then $P_{m}$ is transverse to $\mathcal{F}$. In some open neighborhood $U \subset G$ of $m_{0}$, the leaves of $\mathcal{F}$ intersect $U$ in level sets of a submersion $\Omega: U \rightarrow P_{m_{0}}$. Now let $v=\dot{\gamma}(0) \in T_{m} M$ be the derivative of some path $\gamma$ on $M, m \in M \cap U$. Then, denoting by $A^{\gamma}$ the parallel action along $\gamma$ determined by $D$, we have

$$
\Omega\left(A_{t, 0}^{\gamma}(g)\right)=\Omega(g)
$$

for all $t$ sufficiently close to zero, and all $g \in P_{\gamma(t)}$ sufficiently close to $\gamma(t) \in M$.

If $\left.\mathfrak{g}\right|_{M \cap U}$ denotes the restriction of $\mathfrak{g}$, and $\mathfrak{g}_{0}:=T_{m_{0}} P_{m_{0}}$, then we obtain a map $\omega: \mathfrak{g}_{M \cap U} \rightarrow \mathfrak{g}_{0}$ by differentiating $\Omega: U \rightarrow P_{m_{0}}$ :

$$
\omega\left(\left.\frac{d}{d s} g(s)\right|_{s=0}\right):=\left.\frac{d}{d s} \Omega(g(s))\right|_{s=0} .
$$

As usual, we are viewing an element of $\mathfrak{g}$ as an equivalence class of paths in a source fibre. Applying Theorem 4.1 we calculate

$$
\begin{aligned}
\omega\left(\nabla_{v} X\right) & =\omega\left(\left.\partial_{t} \frac{\partial}{\partial s} A_{t, 0}^{\gamma}\left(\Phi_{X^{\mathrm{R}}}^{s}(\gamma(t))\right)\right|_{s=0, t=0}\right) \\
& \left.\stackrel{\text { by }}{\stackrel{(5.2)}{=}} \partial_{t} \frac{\partial}{\partial s} \Omega\left(A_{t, 0}^{\gamma}\left(\Phi_{X^{\mathrm{R}}}^{s}(\gamma(t))\right)\right)\right|_{s=0, t=0} \\
& \text { by }\left.\stackrel{(5.1)}{=} \partial_{t} \frac{\partial}{\partial s} \Omega\left(\Phi_{X^{\mathrm{R}}}^{s}(\gamma(t))\right)\right|_{s=0, t=0} \\
& =\left.\partial_{t} \omega(X(\gamma(t)))\right|_{t=0}=\langle d(\omega(X)), v\rangle .
\end{aligned}
$$

So $\omega\left(\nabla_{V} X\right)=\mathcal{L}_{V}(\omega(X))$, for any vector field $V$ on $M \cap U$, where $\mathcal{L}$ denotes Lie derivative. For any two vector fields $V_{1}$ and $V_{2}$ on $M \cap U$ we deduce

$$
\omega\left(\operatorname{curv} \nabla\left(V_{1}, V_{2}\right) X\right)=\mathcal{L}_{V_{1}} \mathcal{L}_{V_{2}}(\omega(X))-\mathcal{L}_{V_{2}} \mathcal{L}_{V_{1}}(\omega(X))-\mathcal{L}_{\left[V_{1}, V_{2}\right]}(\omega(X))=0
$$

This shows that $\nabla$ is flat in a neighborhood of the arbitrarily fixed point $m_{0} \in M$.

The remaining sections are devoted to the proof of part (2) of Theorem 1.2.

\subsection{Proof that $G^{D} \subset G$ is a subgroupoid}

This follows immediately from the following lemma (used again in Section 5.4 below):

Lemma 5.1. Let $b_{1}: U_{1} \rightarrow G$ and $b_{2}: U_{2} \rightarrow G$ be two multipliable local bisections integrating $D$. Then their product $b_{1} b_{2}$ integrates $D$.

Proof. Let $m$ be an arbitrary point in $U_{2}$ (the domain of the product $\left.b_{1} b_{2}\right)$ and let $g_{1}=b_{1}(m)$ and $g_{2}=b_{2}(m)$. The arrow $g_{2}$ terminates at some point $m^{\prime}$ in the domain of $b_{1}$. Since $b_{1}$ and $b_{2}$ integrate $D$, the one-jet $S\left(g_{2}\right)$ of a local bisection at $m$ has representative $b_{2}$, while the one-jet $S\left(g_{1}\right)$ of a local bisection at $m^{\prime}$ has representative $b_{1}$. By the definition of the product in $J^{1} G, S\left(g_{1}\right) S\left(g_{2}\right)$ is the one-jet at $m$ of the product $b_{1} b_{2}$, a one-jet that must coincide with $S\left(g_{1} g_{2}\right)$, because $D$ is a Cartan connection. So the image of the tangent map of $b_{1} b_{2}$ at $m$ must be $D\left(g_{1} g_{2}\right)$. Since $m$ was arbitrary, this shows $b_{1} b_{2}$ integrates $D$.

Since the identity bisection lies in $G^{D}, G^{D}$ is wide in $G$.

From now on, we suppose that $\nabla$ is flat. 


\subsection{Proof that $G^{D}$ contains an open neighborhood of $M$}

Our proof that $G^{D} \subset G$ is open will rest on the fact that each point $m \in M$ has an open neighborhood lying in $G^{D}$. In fact, we now prove the following stronger result:

Proposition 5.2. Assume $M$ is simply-connected and that $G$ has simply-connected source-fibres. Then, assuming $\nabla$ is flat, $G^{D}=G$.

We begin by recalling that every Lie algebroid equipped with a flat Cartan connection $\nabla$ is an action algebroid, if $M$ is simply-connected [3] (for generalizations, see [5]):

Lemma 5.3. Let $\mathfrak{g}$ be a Lie algebroid over a simply-connected manifold $M$ supporting a flat Cartan connection $\nabla$. Then the subspace $\mathfrak{g}_{0} \subset \Gamma(\mathfrak{g})$ of $\nabla$-parallel sections is a Lie subalgebra acting on $M$, with the corresponding Lie algebra homomorphism $\xi \mapsto \xi^{\dagger}: \mathfrak{g}_{0} \rightarrow \Gamma(T M)$ being given by $\xi^{\dagger}(m):=\# \xi(m)$. Moreover, the vector bundle morphism $(\xi, m) \rightarrow \xi(m): \mathfrak{g}_{0} \times M \rightarrow \mathfrak{g}$ is a Lie algebroid isomorphism between the action algebroid $\mathfrak{g}_{0} \times M$ and $\mathfrak{g}$.

Proof of Proposition 5.2. Applying the lemma to the Lie algebra $\mathfrak{g}$ of $G$, we obtain a Lie algebra $\mathfrak{g}_{0}$ and a morphism of Lie algebroids $\omega: \mathfrak{g} \rightarrow \mathfrak{g}_{0}$, such that $\omega$ restricted to any fibre $\left.\mathfrak{g}\right|_{m}$ is an isomorphism onto $\mathfrak{g}_{0}$. Since $G$ is source-simply-connected, there exists a Lie groupoid morphism $\Omega: G \rightarrow G_{0}$ integrating $\omega: \mathfrak{g} \rightarrow \mathfrak{g}_{0}$, by Lie II for Lie groupoids (see, e.g., [12]). Here $G_{0}$ is the simply-connected Lie group integrating $\mathfrak{g}_{0}$. Because $\omega$ is a point-wise isomorphism, the restriction of $\Omega$ to any source-fibre of $G$, or any target-fibre of $G$, is a local diffeomorphism. In particular, each level set $\Omega^{-1}\left(g_{0}\right)$ of $\Omega$ is a pseudotransformation in the sense of Section 1.5. Moreover, as $\Omega$ is a morphism of Lie groupoids, it is not hard to see that the foliation of $G$ by connected components of these level sets is a pseudoaction of $G$. The tangent $n$-plane field $D^{\prime}$ is accordingly a Cartan connection on $G$, by Proposition 1.3. Since $D^{\prime}$ is integrable by construction, to prove the proposition, it suffices to show $D=D^{\prime}$.

Now $D=D^{\prime}$ precisely when the corresponding Lie groupoid morphisms

$$
S, S^{\prime}: G \rightarrow J^{1} G
$$

are the same. Since $G$ has connected source-fibres, it actually suffices to show that the derivatives $s, s^{\prime}: \mathfrak{g} \rightarrow J^{1} \mathfrak{g}$ coincide, or equivalently, that the corresponding infinitesimal Cartan connections $\nabla, \nabla^{\prime}$ are the same.

Now $\nabla$ is flat by hypothesis, and $\nabla^{\prime}$ is flat by Theorem $1.2(1)$, already proven above. Since $M$ is simply-connected, to show $\nabla^{\prime}=\nabla$ it is therefore sufficient to show that $\nabla$-parallel sections of $\mathfrak{g}$ are also $\nabla^{\prime}$-parallel. Now $X \in \Gamma(\mathfrak{g})$ is $\nabla$-parallel precisely when $\omega(X)$ is a constant $\xi$. Let $v=\dot{\gamma}(0) \in T_{m} M$ be the derivative of some path $\gamma$ on $M$. Also, let $A^{\gamma}$ denote the parallel action along $\gamma$ defined by the connection $D^{\prime}$. Then, by the definition of $D^{\prime}$, we have

$$
\Omega\left(A_{t, 0}^{\gamma}(g)\right)=\Omega(g)
$$

for all $t$ sufficiently close to 0 and all $g \in \alpha^{-1}(\gamma(t))$ sufficiently close to $\gamma(t) \in M$. On the other hand, applying Theorem 4.1 to the connection $\nabla^{\prime}$, we have

$$
\begin{array}{r}
\omega\left(\nabla_{v}^{\prime} X\right)=\omega\left(\left.\partial_{t} \frac{\partial}{\partial s} A_{t, 0}^{\gamma}\left(\Phi_{X^{\mathrm{R}}}^{s}(\gamma(t))\right)\right|_{s=0, t=0}\right)=\left.\partial_{t} \frac{\partial}{\partial s} \Omega\left(A_{t, 0}^{\gamma}\left(\Phi_{X^{\mathrm{R}}}^{s}(\gamma(t))\right)\right)\right|_{s=0, t=0} \\
\stackrel{\text { by }\left.\stackrel{(5.3)}{=} \partial_{t} \frac{\partial}{\partial s} \Omega\left(\Phi_{X^{\mathrm{R}}}^{s}(\gamma(t))\right)\right|_{s=0, t=0}}{ }=\left.\partial_{t} \omega(X(\gamma(t)))\right|_{t=0}=\left.\partial_{t} \xi\right|_{t=0}=0 .
\end{array}
$$




\subsection{Proof that $G^{D} \subset G$ is open}

To show $G^{D} \subset G$ is open, let $g_{0} \in G^{D}$ be any arrow, beginning at some $m_{0} \in M$. By Section 5.3, $m_{0}$ lies in some open neighborhood of $G$ contained entirely in $G^{D}$. In this neighborhood the connection $D$ is tangent to some foliation $\mathcal{F}$ of the neighborhood.

Using the fact that the foliation $\mathcal{F}$ is locally fibrating, and using the fact that the restriction of the target projection $\beta: G \rightarrow M$ to each leaf of $\mathcal{F}$ is a local diffeomorphism, it follows from the implicit function theorem that we can find a chart $\psi: U \times V \rightarrow G$, for a neighborhood of $m_{0}$ in $G$, simultaneously adapted to the foliation and the target projection $\beta$. Indeed, we may take $U$ to be an open subset of $m_{0}$ in $M, V$ to be an open subset of the target-fibre $\beta^{-1}\left(m_{0}\right)$, and may arrange that: (i) $\beta(\psi(m, p))=m$; and (ii) $\psi(U \times\{p\})$ is an integral manifold of $D$ through $p$, and the image of a local bisection of $G$, for every $p \in V$. See Fig. 3.

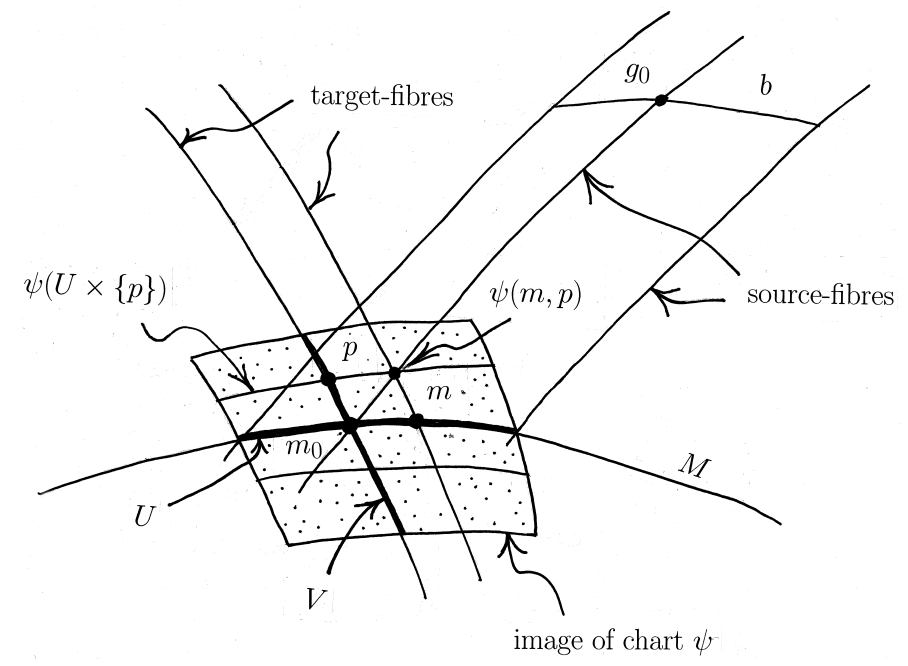

Figure 3. A chart $\psi: U \times V \rightarrow G$ for a neighborhood of $m_{0} \in M$.

Since $g_{0} \in G^{D}$, there is a local bisection $b$ of $G$ integrating $D$ with $b\left(m_{0}\right)=g_{0}$ (see Fig. 3). Shrinking neighborhoods if necessary, we may suppose $b$ has domain $U$. We now use multiplication in the groupoid to translate the chart $\psi$ to a chart $\bar{\psi}: U \times V \rightarrow G$ whose image is a neighborhood of $g_{0}$ : Put $\bar{\psi}(m, p)=b(m) \psi(m, p)$, which is well-defined on account of (i) above. Now $\bar{\psi}$ maps $\{m\} \times V$ diffeomorphically into a fibre of the target projection. On the other hand, for each fixed $p \in V$, the map $m \mapsto \bar{\psi}(m, p): U \rightarrow G$ is an immersion whose image $B_{p}$ is the product of the local bisection $b(U)$ and the local bisection $\psi(U \times\{p\})$ (here viewing local bisections as submanifolds of $G$ ). The two preceding remarks show that $\bar{\psi}$ has a tangent map of full rank, so that $\bar{\psi}(U \times V)$ is an open neighborhood of $g_{0}$. Furthermore, as the local bisection $B_{p}$ is a product of local bisections integrating $D$, for each $p \in V, B_{p}$ is itself an integral manifold of $D$, by Lemma 5.1. Whence $\bar{\psi}(U \times V)$ is an open neighborhood of $g_{0}$ contained in $G^{D}$.

\subsection{Proof that $G^{D} \subset G$ is closed}

That $G^{D} \subset G$ is closed follows from a general property of involutive $n$-plane fields: The torsion tensor $\tau$, a section of $\wedge^{2} D^{*} \otimes(T M / D)$ defined by $\tau\left(V_{1}, V_{2}\right)=\left[V_{1}, V_{2}\right] \bmod D$, must vanish on $G^{D}$. Since $\tau$ is continuous, the complement of $G^{D}$ in $G$ is therefore open.

Since $G^{D}$ is simultaneously open and closed in $G$, it is a union of connected components. Since the identity bisection integrates any Cartan connection $D$ on $G$, we have $G^{0} \subset G$. This completes the proof of part (2) of Theorem 1.2. 


\section{A The canonical Cartan connection for Riemannian geometry}

Here we sketch the construction of the canonical Cartan connection associated with every Riemannian manifold $M$. Details will be given elsewhere. The detailed construction of the corresponding infinitesimal connection appears in [4]. For simplicity, we shall take 'isometry' to mean 'orientation preserving isometry'. For then the transitive Lie groupoid $G \subset J^{1}(M \times M)$ defined in Section 1.2 will have connected isotropy groups and connected source fibres ( $M$ is assumed to be connected).

Define the prolongation $\mathrm{p} G \subset J^{1} G$ of $G$ by $\mathrm{p} G=J^{2}(M \times M) \cap J^{1} G$. Here $J^{2}(M \times M)$ denotes the Lie groupoid of two-jets of local bisections of $M \times M$, regarded as a subgroupoid of $J^{1}\left(J^{1}(M \times M)\right)$.

Lemma A.1. The natural projection $\mathrm{p} G \rightarrow G$ is an isomorphism.

Supposing the lemma to be true, we let $S: G \rightarrow J^{1} G$ be the unique right-inverse for the natural projection $J^{1} G \rightarrow G$ that has $\mathrm{p} G$ as its image. Then $S$ is a Cartan connection defining an $n$-plane field $D$ on $G$.

Proposition A.2. A local transformation $\phi$ of $M$ is an isometry if and only if

$$
\phi(m)=\beta(b(m))
$$

for some local bisection b of $G$ integrating $D$.

Here $\beta: G \rightarrow M$ denotes the target projection.

Proof. The local transformations $\phi$ of $M$ that are isometries are in one-to-one correspondence with the holonomic bisections of $G$ (those bisections $b$ that are of the form $b=J^{1} \phi$, for some bisection $\phi$ of $M \times M)$. In one direction the correspondence is given by first-order extension, $\phi \mapsto J^{1} \phi$. In the other direction we pass from a bisection $b$ to a local transformation $\phi$ using (A.1). But it is a fact that a bisection $b$ of $G$ is holonomic if and only if its extension $J^{1} b$ is a bisection of $\mathrm{p} G$, i.e., if and only if $J_{m}^{1} b \in S(b(m))$ at each $m$ in the domain of $b$. Or, in other words, viewing $b$ as a submanifold of $G$, if and only if $b$ is an integral manifold of $D$.

Proof of Lemma A.1. The corresponding infinitesimal statement is true. That is, the natural projection p $\mathfrak{g} \rightarrow \mathfrak{g}$, where $\mathrm{p} \mathfrak{g} \subset J^{1} \mathfrak{g}$ is defined by $\mathrm{pg}:=J^{2}(T M) \cap J^{1} \mathfrak{g}$, is an isomorphism. This follows from the exactness of a natural sequence

$$
0 \rightarrow \operatorname{ker} \delta \rightarrow \operatorname{pg} \rightarrow \mathfrak{g} \rightarrow \operatorname{coker} \delta
$$

where $\delta: T^{*} M \otimes \mathfrak{h} \rightarrow \wedge^{2} T^{*} M \otimes T M$ is Spencer's coboundary operator and $\mathfrak{h} \subset \mathfrak{g}$ is the kernel of the anchor of $\mathfrak{g}$ (the Lie algebra bundle of skew-symmetric endomorphisms of tangent spaces in the present case). For details see [4].

To show that $\mathrm{p} G \rightarrow G$ is injective it suffices to show that the pre-image $\Sigma \subset \mathrm{p} G$ of the subgroup of identity elements is trivial, i.e., consists only of identity elements. The Lie algebroid of $\Sigma$ is trivial, because it is the kernel of $\mathrm{pg} \rightarrow \mathfrak{g}$. That the totally intransitive Lie groupoid $\Sigma$ itself is trivial follows from the fact that its fibres are simply-connected Abelian Lie groups, as is not too hard to show.

Since $\mathrm{pg} \rightarrow \mathfrak{g}$ is surjective, the morphism $\mathrm{p} G \rightarrow G$ is a submersion. Since its image $I$ is a wide subgroupoid of $G$, this image must be open and closed. Since $G$ has source-connected fibres, $G$ is connected, implying $I=G$. 


\section{References}

[1] Armstrong S., Note on pre-Courant algebroid structures for parabolic geometries, arXiv:0709.0919.

[2] Armstrong S., Lu R., Courant algebroids in parabolic geometry, arXiv:1112.6425.

[3] Blaom A.D., Geometric structures as deformed infinitesimal symmetries, Trans. Amer. Math. Soc. 358 (2006), 3651-3671, math.DG/0404313.

[4] Blaom A.D., Lie algebroids and Cartan's method of equivalence, Trans. Amer. Math. Soc. 364 (2012), 3071-3135, math.DG/0509071.

[5] Blaom A.D., The infinitesimalization and reconstruction of locally homogeneous manifolds, SIGMA 9 (2013), 074, 19 pages, arXiv:1304.7838.

[6] Blaom A.D., Pseudogroups via pseudoactions: unifying local, global, and infinitesimal symmetry, J. Lie Theory 26 (2016), 535-565, arXiv:1410.6981.

[7] Cannas da Silva A., Weinstein A., Geometric models for noncommutative algebras, Berkeley Mathematics Lecture Notes, Vol. 10, Amer. Math. Soc., Providence, RI, Berkeley Center for Pure and Applied Mathematics, Berkeley, CA, 1999.

[8] Čap A., Slovák J., Parabolic geometries. I. Background and general theory, Mathematical Surveys and Monographs, Vol. 154, Amer. Math. Soc., Providence, RI, 2009.

[9] Cartan E., Les systèmes de Pfaff, à cinq variables et les équations aux dérivées partielles du second ordre, Ann. Sci. École Norm. Sup. (3) 27 (1910), 109-192.

[10] Courant T.J., Dirac manifolds, Trans. Amer. Math. Soc. 319 (1990), 631-661.

[11] Crainic M., Differentiable and algebroid cohomology, van Est isomorphisms, and characteristic classes, Comment. Math. Helv. 78 (2003), 681-721, math.DG/0008064.

[12] Crainic M., Fernandes R.L., Lectures on integrability of Lie brackets, in Lectures on Poisson geometry, Geom. Topol. Monogr., Vol. 17, Geom. Topol. Publ., Coventry, 2011, 1-107, math.DG/0611259.

[13] Crainic M., Salazar M.A., Jacobi structures and Spencer operators, J. Math. Pures Appl. 103 (2015), 504521, arXiv:1309.6156.

[14] Crainic M., Salazar M.A., Struchiner I., Multiplicative forms and Spencer operators, Math. Z. 279 (2015), 939-979, arXiv:1210.2277.

[15] Crampin M., Saunders D., Cartan geometries and their symmetries. A Lie algebroid approach, Atlantis Studies in Variational Geometry, Vol. 4, Atlantis Press, Paris, 2016.

[16] del Hoyo M.L., Fernandes R.L., Riemannian metrics on differentiable stacks, arXiv:1601.05616.

[17] Dufour J.P., Zung N.T., Poisson structures and their normal forms, Progress in Mathematics, Vol. 242, Birkhäuser Verlag, Basel, 2005.

[18] Ehresmann C., Les connexions infinitésimales dans un espace fibré différentiable, in Colloque de topologie (espaces fibrés), Bruxelles, 1950, Georges Thone, Liège, Masson et Cie., Paris, 1951, 29-55.

[19] Hitchin N., Generalized Calabi-Yau manifolds, Q. J. Math. 54 (2003), 281-308, math.DG/0209099.

[20] Kirillov A.A., Local Lie algebras, Russ. Math. Surv. 31 (1976), no. 4, 55-76.

[21] Kirillov A.A., Letter to the editors: Correction to "Local Lie algebras" (Russ. Math. Surv. 31 (1976), no. 4, 55-76), Russ. Math. Surv. 32 (1977), no. 1, 268.

[22] Lichnerowicz A., Les variétés de Jacobi et leurs algèbres de Lie associées, J. Math. Pures Appl. 57 (1978), 453-488.

[23] Mackenzie K.C.H., General theory of Lie groupoids and Lie algebroids, London Mathematical Society Lecture Note Series, Vol. 213, Cambridge University Press, Cambridge, 2005.

[24] Morimoto T., Geometric structures on filtered manifolds, Hokkaido Math. J. 22 (1993), $263-347$.

[25] Salazar M.A., Pfaffian groupoids, Ph.D. Thesis, University of Utrecht, The Netherlands, 2013, arXiv:1306.1164.

[26] Sharpe R.W., Differential geometry: Cartan's generalization of Klein's Erlangen program, Graduate Texts in Mathematics, Vol. 166, Springer-Verlag, New York, 1997.

[27] Xu X., Twisted Courant algebroids and coisotropic Cartan geometries, J. Geom. Phys. 82 (2014), 124-131, arXiv:1206.2282. 